\title{
Two-loop massive operator matrix elements for unpolarized heavy flavor production to $O(\epsilon)$
}

\author{
Isabella Bierenbaum ${ }^{\text {a }}$, Johannes Blümlein ${ }^{\mathrm{a}, *}$, \\ Sebastian Klein ${ }^{a}$, Carsten Schneider ${ }^{b}$ \\ a Deutsches Elektronen-Synchrotron, DESY, Platanenallee 6, D-15738 Zeuthen, Germany \\ ${ }^{\mathrm{b}}$ Research Institute for Symbolic Computation (RISC), Johannes Kepler University, \\ Altenbergerstraße 69, A-4040 Linz, Austria
}

Received 3 March 2008; received in revised form 12 May 2008; accepted 21 May 2008

Available online 27 May 2008

\begin{abstract}
We calculate the $O\left(\alpha_{S}^{2}\right)$ massive operator matrix elements for the twist-2 operators, which contribute to the heavy flavor Wilson coefficients in unpolarized deeply inelastic scattering in the region $Q^{2} \gg m^{2}$, up to the $O(\varepsilon)$ contributions. These terms contribute through the renormalization of the $O\left(\alpha_{s}^{3}\right)$ heavy flavor Wilson coefficients of the structure function $F_{2}\left(x, Q^{2}\right)$. The calculation has been performed using light-cone expansion techniques without using the integration-by-parts method. We represent the individual Feynman diagrams by generalized hypergeometric structures, the $\varepsilon$-expansion of which leads to infinite sums depending on the Mellin variable $N$. These sums are finally expressed in terms of nested harmonic sums using the general summation techniques implemented in the Sigma package.
\end{abstract}

(c) 2008 Elsevier B.V. All rights reserved.

\section{Introduction}

The heavy flavor corrections to deeply inelastic scattering constitute an important part of the structure functions in the lower $x$ region, cf. [1, Fig. 2]. The current world data for the nucleon structure functions $F_{2}^{p, d}\left(x, Q^{2}\right)$ reached the precision of a few per cent over a wide kinematic region. Therefore both for the determination of the QCD scale $\Lambda_{\mathrm{QCD}}$ and the detailed shapes

\footnotetext{
* Corresponding author.

E-mail address: johannes.bluemlein@desy.de (J. Blümlein).
} 
of the partonic distribution functions the analysis at the level of the $O\left(\alpha_{s}^{3}\right)$ corrections is required to control the theory-errors on the level of the experimental accuracy and below [2]. In a recent non-singlet analysis [3] errors for $\alpha_{S}\left(M_{Z}^{2}\right)$ of $O(1.5 \%)$ were obtained extending the analysis effectively to $\mathrm{N}^{3} \mathrm{LO}$. In the flavor singlet case the yet unknown 3-loop heavy flavor Wilson coefficients prevent a consistent 3-loop analysis. Due to the large statistics in the lower $x$ region one may hope to eventually improve the accuracy of $\alpha_{s}\left(M_{Z}^{2}\right)$ beyond the above value.

The heavy flavor corrections to $F_{2}^{p, d}\left(x, Q^{2}\right)$ were calculated to 2-loop order in the whole kinematic domain in a semi-analytic way in $x$-space in Refs. [4]. A fast implementation for complex $N$-space was given in [5]. In the range of higher values of $Q^{2}$ one may calculate the heavy flavor Wilson coefficients of the structure functions $F_{2}\left(x, Q^{2}\right)$ and $F_{L}\left(x, Q^{2}\right)$ in analytic form. For $F_{2}\left(x, Q^{2}\right)$ this calculation has been performed to 2-loop order in [6,7] and for $F_{L}\left(x, Q^{2}\right)$ to 3-loop order in [8]. In the region $Q^{2} \gg m^{2}$ the heavy flavor Wilson coefficients for deep-inelastic scattering factorize into massive operator matrix elements $A_{i j}\left(\mu^{2} / \mathrm{m}^{2}\right)$ and the massless Wilson coefficients $C_{k}\left(Q^{2} / \mu^{2}\right)$ [9-11] for all but the power suppressed contributions. The massive operator matrix elements are universal and contain all the mass dependence in the logarithmic orders and the constant term. The process dependence is due to the massless Wilson coefficients. In the case of the structure function $F_{2}\left(x, Q^{2}\right)$ the asymptotic heavy flavor contributions become quantitatively very close to those obtained in the complete calculation $[4,12,13]$ at LO and NLO already for $Q^{2} \gtrsim 10 \mathrm{~m}^{2}$. These scales are sufficiently low and match with the region analyzed in deeply inelastic scattering.

In the present paper we perform a first step towards the 3-loop heavy flavor Wilson coefficients for the structure function $F_{2}\left(x, Q^{2}\right)$. The renormalization of the massive operator matrix elements to 3-loop order encounters also the contributions of $O(\varepsilon)$ at $O\left(\alpha_{s}^{2}\right)$, which have not yet been calculated before. ${ }^{1}$ The 2-loop $O(\varepsilon)$ terms form finite contributions to the $O\left(a_{s}^{3}\right)$, with $a_{s}=\alpha_{s} /(4 \pi)$, matrix elements with the single pole terms emerging at 1 st order. We extend the work presented previously in Ref. [7]. For the calculation of the $O(\varepsilon)$ 2-loop contributions our representation which is based on hypergeometric integrals was extended straightforwardly. However, many more infinite nested sums, which contain the Mellin variable $N$, had to be evaluated for the first time, since other available techniques [16-18] could not be used for this purpose. We applied both suitable integral representations and the summation package Sigma [19], which solves these sums in $\Pi \Sigma$-fields. In the result all sums can be expressed in terms of nested harmonic sums [16,20].

The paper is organized as follows. In Section 2 the structure of the heavy flavor contributions to the deeply inelastic structure function is summarized for the kinematic region $Q^{2} \gg m^{2}$. The renormalization of the massive operator matrix elements to 3-loop order is described in Section 3. In Section 4 the $O(\varepsilon)$ contributions to the 2-loop operator matrix elements are calculated. Section 5 contains the conclusions. In the appendices we present details of the calculation, newly derived infinite sums and related functions depending on the Mellin parameter $N$, and a further check on our result comparing the Abelian part of the first moment with the corresponding part of the on-shell photon propagator.

\footnotetext{
1 In the massless case the off-shell operator matrix elements were calculated too $O\left(\varepsilon^{0}\right)$ for space-like momenta in the $\overline{\mathrm{MS}}$-scheme for unpolarized and polarized deeply inelastic scattering in $[14,15]$, which are needed in the calculation of the 3-loop anomalous dimensions.
} 


\section{Basic formalism}

In the twist-2 approximation, the deep-inelastic nucleon structure functions $F_{n}\left(x, Q^{2}\right)$, $n=2, L$, are described as Mellin convolutions between the parton densities $f_{j}\left(x, \mu^{2}\right)$ and the Wilson coefficients $\mathrm{C}_{i}^{j}\left(x, Q^{2} / \mu^{2}\right), j=q, g$,

$$
F_{n}\left(x, Q^{2}\right)=\sum_{j} \mathrm{C}_{n}^{j}\left(x, \frac{Q^{2}}{\mu^{2}}\right) \otimes f_{j}\left(x, \mu^{2}\right)
$$

to all orders in perturbation theory due to the factorization theorem. Here $\mu^{2}$ denotes the factorization scale and the Mellin convolution is given by the integral

$$
[A \otimes B](x)=\int_{0}^{1} d x_{1} \int_{0}^{1} d x_{2} \delta\left(x-x_{1} x_{2}\right) A\left(x_{1}\right) B\left(x_{2}\right) .
$$

The distributions $f_{j}$ refer to massless partons and the heavy flavor effects are contained in the Wilson coefficients only. As was shown in Ref. [6] in the region $Q^{2} \gg m^{2}$ all non-power contributions to the heavy quark Wilson coefficients obey

$$
H_{n ; i}^{\mathrm{fl}}\left(\frac{Q^{2}}{m^{2}}, \frac{m^{2}}{\mu^{2}}, x\right)=C_{n ; k}^{\mathrm{fl}}\left(\frac{Q^{2}}{\mu^{2}}, x\right) \otimes A_{k, i}^{\mathrm{fl}}\left(\frac{m^{2}}{\mu^{2}}, x\right),
$$

where $C_{n ; k}^{\mathrm{fl}}\left(Q^{2} / \mu^{2}, x\right)$ are the Wilson coefficients for massless partons and $A_{k, i}^{\mathrm{fl}}\left(m^{2} / \mu^{2}, x\right)$ are the massive operator matrix elements. Here $\mu$ refers to the factorization scale between the heavy and light contributions in $\mathrm{C}_{n}^{j}$. In the convolution (3) only those terms are accounted for which contribute to the respective heavy flavor Wilson coefficient functions. The index fl denotes the flavor-decomposition and labels the pure-singlet and gluon contributions (PS,G) and three nonsinglet $\left(\mathrm{NS}^{ \pm}, \mathrm{NS}^{\mathrm{v}}\right)$ combinations. Due to the fact that the diagrams considered here contain one heavy quark line, at $O\left(a_{S}\right)$ only $A_{Q g}$ contributes. Beginning with $O\left(a_{S}^{2}\right)$ there is also the puresinglet $A_{Q q}^{\mathrm{PS}}$ and the non-singlet term $A_{Q q}^{\mathrm{NS}^{+}}$, while at $O\left(a_{s}^{3}\right)$ also the two other non-singlet terms $A_{Q q}^{\mathrm{NS}^{-}}$and $A_{Q q}^{\mathrm{NS}^{\mathrm{v}}}$ contribute. The corresponding combinations of quark distributions for the singlet and non-singlet terms are $\Sigma\left(x, Q^{2}\right)$ and $q^{\mathrm{NS}^{1}}\left(x, Q^{2}\right)$ with

$$
\begin{aligned}
& \Sigma\left(x, Q^{2}\right)=\sum_{k=1}^{N_{l}}\left[q_{k}\left(x, Q^{2}\right)-\bar{q}_{k}\left(x, Q^{2}\right)\right], \\
& q_{m n}^{\mathrm{NS}^{ \pm}}\left(x, Q^{2}\right)=\left[q_{m}\left(x, Q^{2}\right) \pm \bar{q}_{m}\left(x, Q^{2}\right)\right]-\left[q_{n}\left(x, Q^{2}\right) \pm \bar{q}_{n}\left(x, Q^{2}\right)\right], \\
& q^{\mathrm{NS}^{\mathrm{v}}}\left(x, Q^{2}\right)=\sum_{k=1}^{N_{f}}\left[q_{k}\left(x, Q^{2}\right)-\bar{q}_{k}\left(x, Q^{2}\right)\right] .
\end{aligned}
$$

$N_{l}$ denotes the number of light quark flavors. The massless Wilson coefficients were calculated in [9-11] to 3-loop orders. The massive operator matrix elements are process independent quantities. The factorization (3) is a consequence of the renormalization group equation. In Mellin space the operator matrix elements $A_{k, i}^{\mathrm{fl}}$ and light flavor Wilson coefficients obey the following 
expansions:

$$
\begin{aligned}
& A_{k, i}^{\mathrm{fl}}\left(\frac{m^{2}}{\mu^{2}}\right)=\left\langle i\left|O_{k}\right| i\right\rangle=\delta_{k, i}+\sum_{l=1}^{\infty} a_{s}^{l} A_{k, i}^{\mathrm{fl},(l)}, \quad i=q, g, \\
& C_{2 ; i}^{\mathrm{fl}}\left(\frac{Q^{2}}{\mu^{2}}\right)=\delta_{i, q}+\sum_{l=1}^{\infty} a_{s}^{l} C_{2, i}^{\mathrm{fl},(l)}, \quad i=q, g,
\end{aligned}
$$

of the twist-2 flavor singlet, non-singlet and gluon operators $O_{k}^{\mathrm{NS}, S, g}$ between partonic states $|i\rangle$, which are related by collinear factorization to the initial-state nucleon states $|N\rangle$. The local operators are given by

$$
\begin{aligned}
& O_{q, r}^{\mathrm{NS}, \mu_{1}, \ldots, \mu_{N}}(z)=\frac{1}{2} i^{N-1} S\left[\bar{q}(z) \gamma^{\mu_{1}} D^{\mu_{2}} \cdots D^{\mu_{N}} \frac{\lambda_{r}}{2} q(z)\right]-\text { Trace Terms, } \\
& O_{q}^{\mathrm{S}, \mu_{1}, \ldots, \mu_{N}}(z)=\frac{1}{2} i^{N-1} S\left[\bar{q}(z) \gamma^{\mu_{1}} D^{\mu_{2}} \cdots D^{\mu_{N}} q(z)\right]-\text { Trace Terms, } \\
& O_{g}^{\mu_{1}, \ldots, \mu_{N}}(z)=\frac{1}{2} i^{N-2} S\left[F_{\alpha}^{a, \mu_{1}}(z) D^{\mu_{2}} \cdots D^{\mu_{N-1}} F^{a, \alpha, \mu_{N}}(z)\right]-\text { Trace Terms. }
\end{aligned}
$$

Here $S$ denotes the operator which symmetrizes all Lorentz-indices and $D_{\mu_{1}}=\partial_{\mu_{1}}-g t_{a} A_{\mu_{1}}^{a}$ is the covariant derivative, $q(z), \bar{q}(z)$ and $F^{a, \mu v}(z)$ denote the quark-, anti-quark field and the gluon field-strength operators, with $g=\left(4 \pi \alpha_{s}\right)^{1 / 2}=\left(16 \pi^{2} a_{S}\right)^{1 / 2}$ the strong coupling constant, $t_{a}$ the generators of $S U(3)_{c}$, and $\lambda_{r}$ the Gell-Mann matrices of $S U(3)_{F}$. The Feynman rules for the operator insertions are given in [7,21].

Heavy flavor contributions emerge in the diagrams as virtual contributions and massive quark lines in the final state. Whereas the latter emerge only for $W^{2}>\left(2 m_{Q}+m_{p}\right)^{2}$, which introduces a hierarchy of heavy flavor production, the former contributions are present in general.

\section{Renormalization of the matrix elements}

The massive operator matrix elements contain ultraviolet and collinear singularities which have to be renormalized. Charge-, mass-, operator-, and wave function renormalization have to be performed. Collinear singularities appear in those parts of the diagrams with vertices which link only to massless lines, and are specific to the particular classes of diagrams. Since in the present case at least one closed fermion line is massive, collinear singularities appear only at $O\left(a_{s}^{2}\right)$. The un-renormalized massive operator matrix elements read

$$
\hat{\hat{A}}_{i j}=\delta_{i j}+\sum_{k=0}^{\infty} \hat{a}_{s}^{k} \hat{\hat{A}}_{i j}^{(k)} .
$$

Here $\hat{a}_{s}$ denotes the bare coupling constant. To 2-loop order, the corresponding diagrams were given in Ref. [6]. Here one has to distinguish one-particle irreducible and reducible diagrams, which both contribute in the calculation. We would like to remind the reader of the background of this aspect.

If one evaluates the heavy-quark Wilson coefficients in an usual Feynman-diagram calculation, the matrix elements are given by diagrams of the type depicted in Fig. 1. The incoming gluon is factorized from the nucleon, i.e. we assume the parton life-time $\tau_{L}$ being much longer than the interaction time $\tau_{I}$ of the virtual photon with the nucleon. As is well known [22], this 


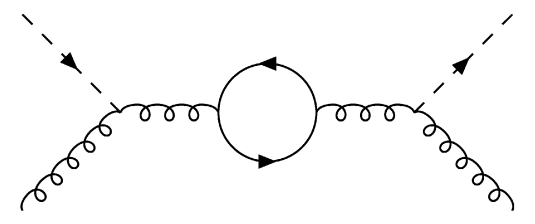

Fig. 1. Massive quark self-energy correction to virtual scalar-gluon scattering.

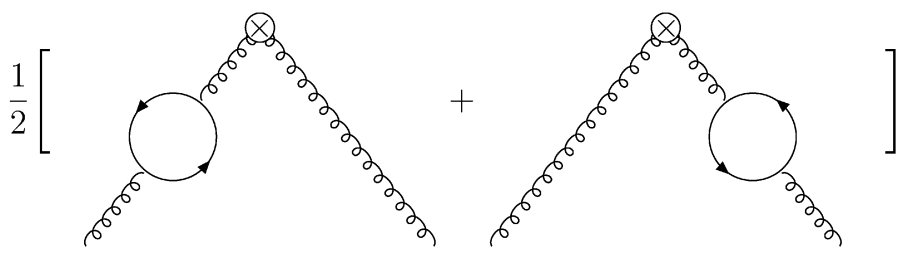

Fig. 2. $O\left(a_{s}\right)$ Self-energy correction due to massive quarks for the operator matrix element $A_{g g}^{(1)}$.

condition is fulfilled whenever $k_{\perp}^{2} \ll Q^{2}$ and neither the Bjorken variable $x$ is very small $(x \ll 1)$ nor large $(x \approx 1)$. This is the case performing the Bjorken limit and applying the collinear parton model, in which the incoming massless partons are dealt with as on-shell particles. Also in this case, self-energy diagrams for the incoming parton lines are present. However, one may factorize these contributions into the non-perturbative parton densities at leading twist, respectively parton correlation functions at higher twist, since these contributions are virtual and are always present whatever hard scattering cross section is considered. One consequence is that at $O\left(a_{s}^{2}\right)$ there are no diagrams with two fermion lines, respectively at $O\left(a_{s}^{3}\right)$ none with three fermion lines in the general heavy flavor Wilson coefficients. However, cf. [4, (3.5), (3.6)], the bare and renormalized parameters of the theory are now related by logarithmic corrections of the type $\propto a_{s}\left(\mu^{2}\right) \sum_{q=4}^{6} \ln \left(m_{q}^{2} / \mu^{2}\right)$, which do not decouple.

The situation is different in case of the operator matrix elements obtained after the light-cone expansion is being performed. Here, the line between the two virtual photon- or weak gauge boson vertices is contracted. This line may contain virtual corrections, see e.g. Fig. 1, which would be lost in the process of contraction. They have to be accounted for in attaching these self-energies to outer lines of the contracted diagram, see Fig. 2. From the case of the fermionfermion anomalous dimension at leading order these aspects are known for long [23-25].

In kinematic regions, where higher twist effects can be safely neglected $[3,26]$ and at sufficiently high scales $Q^{2}$, the scaling violations of deeply inelastic structure functions are due to the running coupling constant and heavy quark mass effects, after target mass effects [27] have been accounted for. We will further assume that we are in a region where power corrections due to heavy quarks are negligibly small, i.e., the heavy quark effects contribute logarithmically $\propto \ln ^{l}\left(m^{2} / \mu^{2}\right), l \geqslant 0$. In this region one may express the structure functions $F_{i}\left(N, Q^{2}\right)$ in Mellin space by

$$
\begin{aligned}
F_{i}\left(N, Q^{2}\right)= & \sum_{l=1}^{N_{l}} C_{i, q}\left(N, Q^{2} / \mu_{f}^{2} ; a_{s}\left(\mu_{r}^{2}\right)\right) \cdot\left[q_{l}\left(N, \mu_{f}^{2} / \mu_{0}^{2}, a_{s}\left(\mu_{r}^{2}\right)\right)\right. \\
& \left.+\bar{q}_{l}\left(N, \mu_{f}^{2} / \mu_{0}^{2}, a_{s}\left(\mu_{r}^{2}\right)\right)\right] \\
& +C_{i, g}\left(N, Q^{2} / \mu_{f}^{2} ; a_{s}\left(\mu_{r}^{2}\right)\right) \cdot g\left(N, \mu_{f}^{2} / \mu_{0}^{2}, a_{s}\left(\mu_{r}^{2}\right)\right)
\end{aligned}
$$




$$
\begin{aligned}
& +\sum_{h=1}^{N_{l}} H_{i, q}\left(N, Q^{2} / \mu_{f}^{2} ; a_{s}\left(\mu_{r}^{2}\right)\right) \cdot\left[q_{l}\left(N, \mu_{f}^{2} / \mu_{0}^{2}, a_{s}\left(\mu_{r}^{2}\right)\right)\right. \\
& \left.+\bar{q}_{l}\left(N, \mu_{f}^{2} / \mu_{0}^{2}, a_{s}\left(\mu_{r}^{2}\right)\right)\right] \\
& +H_{i, g}\left(N, Q^{2} / \mu_{f}^{2} ; a_{s}\left(\mu_{r}^{2}\right)\right) \cdot g\left(N, \mu_{f}^{2} / \mu_{0}^{2}, a_{s}\left(\mu_{r}^{2}\right)\right)
\end{aligned}
$$

in the fixed flavor scheme [6,7,28], which we adopt in the following. ${ }^{2}$ Here $\mu_{r}$ and $\mu_{f}$ denote the renormalization and factorization scales, respectively, $\mu_{0}$ is a hadronic scale, and $q, \bar{q}$ and $g$ denote the quark- and gluon distribution functions. Furthermore, the heavy quark Wilson coefficients factorize according to (3), which is described by the scale $\mu$. In the following we identify all these scales $\mu=\mu_{r}=\mu_{f}$. Since the structure functions $F_{i}\left(N, Q^{2}\right)$ do not depend on these scales they obey the following renormalization group equation (RGE) [31]

$$
\left[\mathcal{D}+2 \gamma_{j}\right] F_{n}\left(N, Q^{2}\right)=0,
$$

where the differential operator $\mathcal{D}$ is defined by

$$
\mathcal{D}=\mu^{2} \frac{\partial}{\partial \mu^{2}}+\beta\left(a_{s}\left(\mu^{2}\right)\right) \frac{\partial}{\partial a_{s}\left(\mu^{2}\right)}-\gamma_{m}\left(a_{s}\left(\mu^{2}\right)\right) m^{2}\left(\mu^{2}\right) \frac{\partial}{\partial m^{2}\left(\mu^{2}\right)} .
$$

$\beta\left(a_{s}\right)$ denotes the $\beta$-function, $\gamma_{m}\left(a_{s}\right)$ the mass anomalous dimension, and $\gamma_{j}$ denote the anomalous dimensions of the quark fields. Here we discuss the case of conserved currents, which have vanishing anomalous dimensions. ${ }^{3}$

The RGE for the Wilson coefficients and the parton distributions read [24,33]

$$
\begin{aligned}
& {\left[\mathcal{D} \delta_{k j}-\gamma_{k j}^{N}\left(a_{s}\right)\right] \mathrm{C}_{n}^{j}\left(N, Q^{2} / \mu^{2}\right)=0,} \\
& {\left[\left(\mathcal{D}+2 \gamma_{j}\right) \delta_{k k^{\prime}}+\gamma_{k k^{\prime}}^{N}\left(a_{s}\right)\right] f_{j k^{\prime}}\left(N, \mu^{2} / \mu_{0}^{2}\right)=0,}
\end{aligned}
$$

with

$$
f_{j k}\left(N, \mu^{2} / \mu_{0}^{2}\right)=\left\langle j\left|O_{k}\right| j\right\rangle .
$$

In the following we describe the renormalization to $O\left(a_{s}^{3}\right)$.

\subsection{Charge renormalization}

We perform the charge renormalization in the $\overline{\mathrm{MS}}$-scheme. This allows to compare the results obtained in the QCD analysis of deeply inelastic scattering data with analyzes of other data. The bare coupling constant $\hat{a}_{s}$ is expressed by the renormalized coupling $a_{s}$ in the $\overline{\mathrm{MS}}$ scheme by

$$
\begin{aligned}
\hat{a}_{s}(\varepsilon) & =Z_{g}^{2}\left(\varepsilon, \mu^{2}\right) a_{s}\left(\mu^{2}\right) \\
& =a_{s}\left(\mu^{2}\right)\left[1+\delta a_{s, 1} a_{s}\left(\mu^{2}\right)+\delta a_{s, 2} a_{s}^{2}\left(\mu^{2}\right)\right]+O\left(a_{s}^{4}\right), \\
\delta a_{s, 1} & =S_{\varepsilon} \frac{2 \beta_{0}}{\varepsilon} \\
\delta a_{s, 2} & =S_{\varepsilon}^{2}\left[\frac{4 \beta_{0}^{2}}{\varepsilon^{2}}+\frac{\beta_{1}}{\varepsilon}\right],
\end{aligned}
$$

\footnotetext{
2 Variable flavor schemes are discussed elsewhere [29,30].

3 Calculating the evolution of the transversity structure function $h_{1}\left(x, Q^{2}\right)$ using the forward Compton amplitude, this is not the case, cf. [32].
} 
with $Z_{g}$ the $Z$-factor for the strong charge. Here the spherical factor $S_{\varepsilon}$ is given by

$$
S_{\varepsilon}=\exp \left[\frac{\varepsilon}{2}\left(\gamma_{E}-\ln (4 \pi)\right)\right],
$$

with $\gamma_{E}$ the Euler-Mascheroni number, $\varepsilon=D-4$, and $D$ the dimension of space-time. $\beta_{0}$ and $\beta_{1}$ [34] denote the first expansion coefficients of the $\beta$-function in the massless case

$$
\begin{aligned}
& \frac{d a_{s}\left(\mu^{2}\right)}{d \ln \left(\mu^{2}\right)}=\frac{1}{2} \varepsilon a_{s}\left(\mu^{2}\right)-\sum_{k=0}^{\infty} \beta_{k} a_{s}^{k+2}\left(\mu^{2}\right), \\
& \beta_{0}=\frac{11}{3} C_{A}-\frac{4}{3} T_{F} n_{f}, \\
& \beta_{1}=\frac{34}{3} C_{A}^{2}-4\left(\frac{5}{3} C_{A}+C_{F}\right) T_{F} n_{f} .
\end{aligned}
$$

The color factors for $S U(3)_{c}$ are $C_{F}=\left(N_{c}^{2}-1\right) /\left(2 N_{c}\right)=4 / 3, C_{A}=N_{c}=3, T_{F}=1 / 2 . n_{f}$ denotes the number of active flavors. The renormalized coupling constant is obtained absorbing $Z_{g}$ into the bare coupling $\hat{g}$.

Although in the final result all parameters of the theory shall be dealt with using the $\overline{\mathrm{MS}}$ prescription, in intermediate steps the calculation requires to deal with explicit heavy quark corrections. This was outlined in [6] in case of the strong coupling constant. As we will fix the gauge the only other parameters receiving corrections of this type are the heavy quark masses. In the present context this occurs first at 3-loop order. Due to the presence of mass logarithms these are MOM-scheme expressions. ${ }^{4}$ We do not take the attitude to solve the evolution equations both for the strong coupling and the masses at this stage, however, since (i) the direct comparison of these parameters as extracted from other measurements will not be possible; (ii) we could not match the massive operator matrix elements with the massless $\overline{\mathrm{MS}}$ Wilson coefficients to massive Wilson coefficients. In the final description one maps back to the $\overline{\mathrm{MS}}$ scheme finally using the decoupling formalism [12,39]. This step was not yet rigorously performed in [6].

\subsection{Mass renormalization}

We choose the on-mass-shell scheme for quarks. In case of the heavy quarks the bare mass $\hat{m}$ is related to the renormalized mass by

$$
\begin{aligned}
\hat{m}=m & +\hat{a}_{s} \delta m_{1}+\hat{a}_{s}^{2} \delta m_{2}+O\left(a_{s}^{3}\right), \\
\delta m_{1}= & C_{F} S_{\varepsilon} m\left(\frac{m^{2}}{\mu^{2}}\right)^{\varepsilon / 2}\left[\frac{6}{\varepsilon}-4+\left(4+\frac{3}{4} \zeta_{2}\right) \varepsilon\right], \\
\delta m_{2}= & C_{F} S_{\varepsilon}^{2} m\left(\frac{m^{2}}{\mu^{2}}\right)^{\varepsilon}\left[\frac{1}{\varepsilon^{2}}\left(18 C_{F}+22 C_{A}-8 T_{F}\left(N_{l}+N_{h}\right)\right)\right. \\
& +\frac{1}{\varepsilon}\left(-\frac{45}{2} C_{F}+\frac{91}{2} C_{A}-14 T_{F}\left(N_{l}+N_{h}\right)\right)+C_{F}\left(\frac{199}{8}-\frac{51}{2} \zeta_{2}\right.
\end{aligned}
$$

\footnotetext{
4 To maintain the Slavnov-Taylor identities of QCD in the case of MOM-schemes [35] the calculation has to be performed using the background-field method [36] in [37]. Earlier calculations [27,38] illustrated this reporting different expressions for $Z_{g}$ depending on the vertex considered.
} 


$$
\begin{aligned}
& \left.+48 \ln (2) \zeta_{2}-12 \zeta_{3}\right)+C_{A}\left(-\frac{605}{8}+\frac{5}{2} \zeta_{2}-24 \ln (2) \zeta_{2}+6 \zeta_{3}\right) \\
& \left.+T_{F}\left[N_{l}\left(\frac{45}{2}+10 \zeta_{2}\right)+N_{h}\left(\frac{69}{2}-14 \zeta_{2}\right)\right]\right],
\end{aligned}
$$

(27) is easily obtained. The pole terms to (28) were given in [40], after charge renormalization, and the constant term in [41], see also [42]. The 3-loop corrections were given in [43]. The renormalized mass is obtained absorbing $Z_{m}$ into the bare mass $\hat{m}$. Heavy quark mass effects occur also for massless quark self-energies, see Section 3.4.

\subsection{Operator renormalization}

The local operators which emerge in the light cone expansion contain ultraviolet divergences. These are renormalized by the following $Z$-factors for the flavor non-singlet (NS), singlet (S), and pure singlet (PS) contributions. The formulae are partly generic and have to be adapted, as the diagrams contain at least one heavy quark line, specific color factors need to be projected for the three flavor non-singlet contributions, etc. Here we suppress the argument $N$ in the anomalous dimensions $\gamma_{i j, k}$.

$$
\begin{aligned}
& Z_{\mathrm{NS}}\left(N, a_{s}, \varepsilon\right) \\
& =1+a_{s} S_{\varepsilon} \frac{\gamma_{\mathrm{NS}, 0}}{\varepsilon}+a_{s}^{2} S_{\varepsilon}^{2}\left[\frac{1}{\varepsilon^{2}}\left(\frac{1}{2} \gamma_{\mathrm{NS}, 0}^{2}+\beta_{0} \gamma_{\mathrm{NS}, 0}\right)+\frac{1}{2 \varepsilon} \gamma_{\mathrm{NS}, 1}\right] \\
& +a_{s}^{3} S_{\varepsilon}^{3}\left[\frac{1}{\varepsilon^{3}}\left(\frac{1}{6} \gamma_{\mathrm{NS}, 0}^{3}+\beta_{0} \gamma_{\mathrm{NS}, 0}^{2}+\frac{4}{3} \beta_{0}^{2} \gamma_{\mathrm{NS}, 0}\right)\right. \\
& \left.+\frac{1}{\varepsilon^{2}}\left(\frac{1}{2} \gamma_{\mathrm{NS}, 0} \gamma_{\mathrm{NS}, 1}+\frac{2}{3} \beta_{0} \gamma_{\mathrm{NS}, 1}+\frac{2}{3} \beta_{1} \gamma_{\mathrm{NS}, 0}\right)+\frac{1}{3 \varepsilon} \gamma_{\mathrm{NS}, 2}\right], \\
& Z_{q q}\left(N, a_{s}, \varepsilon\right) \\
& =1+a_{s} S_{\varepsilon} \frac{\gamma_{q q, 0}}{\varepsilon}+a_{s}^{2} S_{\varepsilon}^{2}\left\{\frac{1}{\varepsilon^{2}}\left[\frac{1}{2}\left(\gamma_{q q, 0}^{2}+\gamma_{q g, 0} \gamma_{g q, 0}\right)+\beta_{0} \gamma_{q q, 0}\right]+\frac{1}{2 \varepsilon} \gamma_{q q, 1}\right\} \\
& +a_{s}^{3} S_{\varepsilon}^{3}\left\{\frac { 1 } { \varepsilon ^ { 3 } } \left[\frac{1}{6}\left(\gamma_{q q, 0}^{3}+2 \gamma_{q q, 0} \gamma_{q g, 0} \gamma_{g q, 0}+\gamma_{q g, 0} \gamma_{g g, 0} \gamma_{g q, 0}\right)\right.\right. \\
& \left.+\beta_{0}\left(\gamma_{q q, 0}^{2}+\gamma_{q g, 0} \gamma_{g q, 0}\right)+\frac{4}{3} \beta_{0}^{2} \gamma_{q q, 0}\right]+\frac{1}{\varepsilon^{2}}\left[\frac{1}{2} \gamma_{q q, 0} \gamma_{q q, 1}+\frac{1}{3} \gamma_{q g, 0} \gamma_{g q, 1}\right. \\
& \left.\left.+\frac{1}{6} \gamma_{q g, 1} \gamma_{g q, 0}+\frac{2}{3}\left(\beta_{0} \gamma_{q q, 1}+\beta_{1} \gamma_{q q, 0}\right)\right]+\frac{\gamma_{q q, 2}}{3 \varepsilon}\right\} \\
& Z_{q g}\left(N, a_{s}, \varepsilon\right) \\
& =a_{s} S_{\varepsilon} \frac{\gamma_{q g}, 0}{\varepsilon}+a_{s}^{2} S_{\varepsilon}^{2}\left\{\frac{1}{\varepsilon^{2}}\left[\frac{1}{2}\left(\gamma_{q g, 0} \gamma_{g g, 0}+\gamma_{q q, 0} \gamma_{q g, 0}\right)+\beta_{0} \gamma_{q g, 0}\right]+\frac{1}{2 \varepsilon} \gamma_{q g, 1}\right\} \\
& +a_{s}^{3} S_{\varepsilon}^{3}\left\{\frac { 1 } { \varepsilon ^ { 3 } } \left[\frac{1}{6}\left(\gamma_{q g, 0} \gamma_{g g, 0}^{2}+\gamma_{q q, 0} \gamma_{q g, 0} \gamma_{g g, 0}+\gamma_{q g, 0} \gamma_{g q, 0} \gamma_{q g, 0}+\gamma_{q g, 0} \gamma_{q q, 0}^{2}\right)\right.\right. \\
& \left.+\beta_{0}\left(\gamma_{q g, 0} \gamma_{g g, 0}+\gamma_{q q, 0} \gamma_{q g, 0}\right)+\frac{4}{3} \beta_{0}^{2} \gamma_{q g, 0}\right]+\frac{1}{\varepsilon^{2}}\left[\frac { 1 } { 6 } \left(\gamma_{q g, 1} \gamma_{g g, 0}+\gamma_{q q, 1} \gamma_{q g, 0}\right.\right. \\
& \left.\left.\left.+2 \gamma_{q q, 0} \gamma_{q g, 1}+2 \gamma_{q g, 0} \gamma_{g g, 1}\right)+\frac{2}{3}\left(\beta_{0} \gamma_{q g, 1}+\beta_{1} \gamma_{q g, 0}\right)\right]+\frac{\gamma_{q g, 2}}{3 \varepsilon}\right\}
\end{aligned}
$$




$$
\begin{aligned}
Z_{g q}( & \left.N, a_{s}, \varepsilon\right) \\
= & a_{s} S_{\varepsilon} \frac{\gamma_{g q, 0}}{\varepsilon}+a_{s}^{2} S_{\varepsilon}^{2}\left\{\frac{1}{\varepsilon^{2}}\left[\frac{1}{2}\left(\gamma_{g q, 0} \gamma_{q q, 0}+\gamma_{g g, 0} \gamma_{g q, 0}\right)+\beta_{0} \gamma_{g q, 0}\right]+\frac{1}{2 \varepsilon} \gamma_{g q, 1}\right\} \\
& +a_{s}^{3} S_{\varepsilon}^{3}\left\{\frac { 1 } { \varepsilon ^ { 3 } } \left[\frac{1}{6}\left(\gamma_{g q, 0} \gamma_{q q, 0}^{2}+\gamma_{g g, 0} \gamma_{g q, 0} \gamma_{q q, 0}+\gamma_{g q, 0} \gamma_{q g, 0} \gamma_{g q, 0}+\gamma_{g q, 0} \gamma_{g g, 0}^{2}\right)\right.\right. \\
& \left.+\beta_{0}\left(\gamma_{g q, 0} \gamma_{q q, 0}+\gamma_{g g, 0} \gamma_{g q, 0}\right)+\frac{4}{3} \beta_{0}^{2} \gamma_{g q, 0}\right]+\frac{1}{\varepsilon^{2}}\left[\frac { 1 } { 6 } \left(\gamma_{g q, 1} \gamma_{q q, 0}+\gamma_{g g, 1} \gamma_{g q, 0}\right.\right. \\
& \left.\left.\left.+2 \gamma_{g g, 0} \gamma_{g q, 1}+2 \gamma_{g q, 0} \gamma_{q q, 1}\right)+\frac{2}{3}\left(\beta_{0} \gamma_{g q, 1}+\beta_{1} \gamma_{g q, 0}\right)\right]+\frac{\gamma_{g q, 2}}{3 \varepsilon}\right\}, \\
Z_{g g}( & \left., a_{s}, \varepsilon\right) \\
= & +a_{s} S_{\varepsilon} \frac{\gamma_{g g, 0}}{\varepsilon}+a_{s}^{2} S_{\varepsilon}^{2}\left\{\frac{1}{\varepsilon^{2}}\left[\frac{1}{2}\left(\gamma_{g g, 0}^{2}+\gamma_{g q, 0} \gamma_{q g, 0}\right)+\beta_{0} \gamma_{g g, 0}\right]+\frac{1}{2 \varepsilon} \gamma_{g g, 1}\right\} \\
& +a_{s}^{3} S_{\varepsilon}^{3}\left\{\frac { 1 } { \varepsilon ^ { 3 } } \left[\frac{1}{6}\left(\gamma_{g g, 0}^{3}+2 \gamma_{g g, 0} \gamma_{g q, 0} \gamma_{q g, 0}+\gamma_{g q, 0} \gamma_{q g, 0} \gamma_{q q, 0}\right)\right.\right. \\
& \left.+\beta_{0}\left(\gamma_{g g, 0}^{2}+\gamma_{g q, 0} \gamma_{q g, 0}\right)+\frac{4}{3} \beta_{0}^{2} \gamma_{g g, 0}\right]+\frac{1}{\varepsilon^{2}}\left[\frac{1}{2} \gamma_{g g, 0} \gamma_{g g, 1}+\frac{1}{3} \gamma_{g q, 0} \gamma_{q g, 1}\right. \\
& \left.\left.+\frac{1}{6} \gamma_{g q, 1} \gamma_{q g, 0}+\frac{2}{3}\left(\beta_{0} \gamma_{g g, 1}+\beta_{1} \gamma_{g g, 0}\right)\right]+\frac{\gamma_{g g, 2}}{3 \varepsilon}\right\} .
\end{aligned}
$$

The pure-singlet operator has the following $Z$-factor.

$$
\begin{aligned}
Z_{q q}^{\mathrm{PS}}\left(N, a_{s}, \varepsilon\right) \\
=a_{s}^{2} S_{\varepsilon}^{2}\left\{\frac{1}{2 \varepsilon^{2}} \gamma_{q g, 0} \gamma_{g q, 0}+\frac{1}{2 \varepsilon} \gamma_{q q, 1}^{\mathrm{PS}}\right\} \\
\quad+a_{s}^{3} S_{\varepsilon}^{3}\left\{\frac{1}{\varepsilon^{3}}\left[\frac{1}{6}\left(2 \gamma_{q q, 0} \gamma_{q g, 0} \gamma_{g q, 0}+\gamma_{q g, 0} \gamma_{g g, 0} \gamma_{g q, 0}\right)+\beta_{0} \gamma_{q g, 0} \gamma_{g q, 0}\right]\right. \\
\left.\quad+\frac{1}{\varepsilon^{2}}\left[\frac{1}{2} \gamma_{q q, 0} \gamma_{q q, 1}^{\mathrm{PS}}+\frac{1}{3} \gamma_{q g, 0} \gamma_{g q, 1}+\frac{1}{6} \gamma_{q g, 1} \gamma_{g q, 0}+\frac{2}{3} \beta_{0} \gamma_{q q, 1}^{\mathrm{PS}}\right]+\frac{\gamma_{q q, 2}^{\mathrm{PS}}}{3 \varepsilon}\right\} .
\end{aligned}
$$

The anomalous dimensions $\gamma_{i j, k}(N)$ are related to the splitting functions by

$$
\gamma_{i j, k}(N)=-\int_{0}^{1} d z z^{N-1} P_{i j}^{(k)}(z) .
$$

The renormalized operators are obtained absorbing $Z_{\mathrm{NS}}, Z_{i j, \mathrm{~S}}$, and $Z_{q q}^{\mathrm{PS}}$, into the bare operators, respectively operator matrix elements.

\subsection{Wave function renormalization}

The external legs of the operator matrix elements are treated on-shell to be able to apply their factorization from the nucleon wave functions in the light cone expansion as outlined above. Here the mass scale is set by a heavy quark mass. To the operator matrix elements also one- 
particle reducible diagrams contribute. If either the self-energy insertion on the external legs or the remainder diagram contain only massless lines, with the exception of the tree-level terms, the diagrams are vanishing since one of the factors has no scale. I.e. finite contributions are due to the self-energy insertions containing a massive line. The corresponding corrections are due to the massive contributions to the massless quark self-energy up to 3-loop order and the gluon self-energies up to 2-loop order. The former terms emerge in case of the flavor non-singlet terms $A_{q q, Q}^{\mathrm{NS},(3)}$ and the latter in $A_{Q g}^{(3)}$, while the pure singlet contributions $A_{Q q}^{\mathrm{PS},(3)}$ obtain no corrections.

\subsubsection{Massless external quark lines}

The 2-loop correction reads

$$
\Sigma_{i j}^{(2)}=a_{s}^{2} \delta_{i j} T_{F} C_{F}\left(\frac{m^{2}}{\mu^{2}}\right)^{\varepsilon} S_{\varepsilon}^{2}\left\{\frac{2}{\varepsilon}+\frac{5}{6}+\left[\frac{89}{72}+\frac{\zeta_{2}}{2}\right] \varepsilon+O\left(\varepsilon^{2}\right)\right\} \cdot i \not p .
$$

At $O\left(a_{s}^{2}\right)$ this contribution implies, that the 1st moment of the non-singlet operator matrix element vanishes.

\subsubsection{External gluon lines}

The gluon vacuum polarization is given by

$$
\Pi_{\mu \nu}^{a b}(q)=\left[-g_{\mu \nu} q^{2}+q_{\mu} q_{\nu}\right] \Pi^{a b}\left(q^{2}\right),
$$

with $a$ and $b$ the color indices. The 1-loop and 2-loop corrections read

$$
\begin{aligned}
\Pi_{(1)}^{a b}(0)= & -i a_{s} \delta^{a b} T_{F} S_{\varepsilon}\left(\frac{\hat{m}^{2}}{\mu^{2}}\right)^{\varepsilon / 2} \frac{4}{3}\left\{\frac{2}{\varepsilon}+\frac{\varepsilon}{4} \zeta_{2}+O\left(\varepsilon^{2}\right)\right\}, \\
\Pi_{(2)}^{a b}(0)= & -i a_{s}^{2} \delta^{a b} T_{F} S_{\varepsilon}^{2}\left(\frac{\hat{m}^{2}}{\mu^{2}}\right)^{\varepsilon}\left\{C_{F}\left[\frac{12}{\varepsilon}+\frac{13}{3}+\left(\frac{35}{12}+3 \zeta_{2}\right) \varepsilon\right]\right. \\
& \left.+C_{A}\left[\frac{4}{\varepsilon^{2}}-\frac{5}{\varepsilon}-\left(\frac{13}{12}-\zeta_{2}\right)-\left(\frac{169}{144}+\frac{5}{4} \zeta_{2}-\frac{\zeta_{3}}{3}\right) \varepsilon\right]+O\left(\varepsilon^{2}\right)\right\} .
\end{aligned}
$$

The $C_{F}$-term can be compared with a corresponding contribution in the photon propagator, (C.5), before mass renormalization. At 2-loop order the diagrams $u$ and $v$ from [6] and the term $Z_{q g}^{-1,(1)} \hat{A}_{g g}^{(1)}$ combine in the renormalization to a term which intermediary is absorbed into the strong coupling constant. $a_{s}$ then contains logarithms due to the heavy quark masses, but the $\beta$-function is free of these terms. As mentioned before, the decoupling formalism finally allows to get back to the $\overline{\mathrm{MS}}$ prescription on the expense of changing $\Lambda_{\mathrm{QCD}}$.

\subsection{Mass factorization}

The mass singularities are factored into the functions $\Gamma_{\mathrm{NS}}, \Gamma_{i j, \mathrm{~S}}$ and $\Gamma_{q q, \mathrm{PS}}$, respectively. If all quarks were massless these functions were given by

$$
\begin{aligned}
& \Gamma_{\mathrm{NS}}=Z_{\mathrm{NS}}^{-1}, \\
& \Gamma_{i j, \mathrm{~S}}=Z_{i j, \mathrm{~S}}^{-1}, \\
& \Gamma_{q q, \mathrm{PS}}=Z_{q q, \mathrm{PS}}^{-1},
\end{aligned}
$$


with

$$
\begin{aligned}
& \Gamma_{\mathrm{NS}}\left(N, a_{s}, \varepsilon\right)=1-a_{s} S_{\varepsilon} \frac{\gamma_{\mathrm{NS}, 0}}{\varepsilon}+a_{s}^{2} S_{\varepsilon}^{2}\left[\frac{1}{\varepsilon^{2}}\left(\frac{1}{2} \gamma_{N S, 0}^{2}-\beta_{0} \gamma_{\mathrm{NS}, 0}\right)-\frac{1}{2 \varepsilon} \gamma_{\mathrm{NS}, 1}\right], \\
& \Gamma_{i j, \mathrm{~S}}\left(N, a_{s}, \varepsilon\right)=\delta_{i j}-a_{s} S_{\varepsilon} \frac{\gamma_{i j, 0}}{\varepsilon}+a_{s}^{2} S_{\varepsilon}^{2}\left[\frac{1}{\varepsilon^{2}}\left(\frac{1}{2} \gamma_{i k, 0} \gamma_{k j, 0}-\beta_{0} \gamma_{i j, 0}\right)-\frac{1}{2 \varepsilon} \gamma_{i j, 1}\right], \\
& \Gamma_{q q, \mathrm{PS}}\left(N, a_{s}, \varepsilon\right)=-a_{s}^{2} S_{\varepsilon}^{2}\left[-\frac{1}{2 \varepsilon^{2}} \gamma_{q g, 0} \gamma_{g q, 0}+\frac{1}{2 \varepsilon} \gamma_{q q, \mathrm{PS}, 1}\right] .
\end{aligned}
$$

In the present calculation at least one quark line is massive in each diagram. Therefore the $\Gamma$ matrices (43)-(45) apply to the parts of the diagrams which contain massless lines only, which are at most 2-loop sub-graphs. The mass factorization is therefore different in various sub-classes of contributing Feynman diagrams. The functions $\Gamma_{\mathrm{NS}}, \Gamma_{i j, \mathrm{~S}}$, and $\Gamma_{q q \text {, PS }}$ do thus enter the renormalization of the operator matrix elements only in products with other functions. The singularities contained in $\Gamma_{\mathrm{NS}}, \Gamma_{i j, \mathrm{~S}}$, and $\Gamma_{q q, \mathrm{PS}}$ are absorbed into the bare parton densities, which become scale-dependent in this way.

\subsection{The renormalized operator matrix elements}

The operator matrix element reads after charge and mass renormalization

$$
\begin{aligned}
\hat{A}_{i j}= & \delta_{i j}+a_{s} \hat{\hat{A}}_{i j}^{(1)}+a_{s}^{2}\left[\hat{\hat{A}}_{i j}^{(2)}+\delta m_{1} \frac{d}{d m} \hat{\hat{A}}_{i j}^{(1)}+\delta a_{s}^{(1)} \hat{\hat{A}}_{i j}^{(1)}\right] \\
& +a_{s}^{3}\left[\hat{\hat{A}}_{i j}^{(3)}+\delta a_{s}^{(2)} \hat{\hat{A}}_{i j}^{(1)}+2 \delta a_{s}^{(1)}\left(\hat{\hat{A}}_{i j}^{(2)}+\delta m_{1} \frac{d}{d m} \hat{\hat{A}}_{i j}^{(1)}\right)+\delta m_{1} \frac{d}{d m} \hat{\hat{A}}_{i j}^{(2)}\right. \\
& \left.+\delta m_{2} \frac{d}{d m} \hat{\hat{A}}_{i j}^{(1)}+\delta m_{1}^{2} \frac{1}{2} \frac{d^{2}}{d m^{2}} \hat{\hat{A}}_{i j}^{(1)}\right] .
\end{aligned}
$$

The renormalized operator matrix elements are obtained removing the ultraviolet singularities and collinear singularities of the operator matrix elements,

$$
A_{i j}=Z_{i k}^{-1} \hat{A}_{k l} \Gamma_{l j}^{-1}=\delta_{i j}+a_{s} A_{i j}^{(1)}+a_{s}^{2} A_{i j}^{(2)}+a_{s}^{3} A_{i j}^{(3)} .
$$

Here self energy insertions containing massive lines in the external legs of the operator matrix elements have to be kept.

\section{The $O(\varepsilon)$ contributions}

The $O(\varepsilon)$ contributions to $A_{Q g}, A_{Q q}^{\mathrm{PS}}$ and $A_{q q, Q}^{\mathrm{NS}}$ at $O\left(a_{s}^{2}\right)$ contribute to these quantities at $O\left(a_{s}^{3}\right)$ in combination with the various single pole terms emerging at 1-loop, as outlined in Section 3. The diagrams to be evaluated are shown in [6,7]. The results for the individual unrenormalized diagrams in $O(\varepsilon)$ are given in Appendix A. As outlined before in Ref. [7], we calculate the massive operator matrix elements performing the Feynman-parameter integrals directly, i.e., without using the integration-by-parts method [44] which was applied in [6] up to the terms $O\left(\varepsilon^{0}\right)$ before. We obtain representations in terms of generalized hypergeometric functions [45], which may be expanded to the desired order in $\varepsilon$. With increasing depth in $\varepsilon$, more and more involved nested infinite sums are obtained, which depend on the Mellin-parameter $N$. These sums can be summed applying analytic methods, as integral representations, and general 
summation methods, as encoded in the Sigma package [19]. We applied both methods to evaluate the sums which emerge at $O(\varepsilon)$. The underlying algorithms of Sigma are based on a refined version [46] of Karr's difference field theory of $\Pi \Sigma$-fields [47]. In this algebraic setting one can represent completely algorithmically indefinite nested sums and products without introducing any algebraic relations between them. Note that this general class of sum expressions covers as special cases, e.g., the harmonic sums [16,20] or generalized nested harmonic sums cf. [48-51]. Given such an optimal representation, by introducing as less sums as possible, various summation principles are available in Sigma. In this article we applied the following strategy which has been generalized from the hypergeometric case [52] to the $\Pi \Sigma$-field setting.

1. Given a definite sum that involves an extra parameter $N$, for typical sums see Appendix B. We compute a recurrence relation in $N$ that is fulfilled by the input sum. The underlying difference field algorithms exploit Zeilberger's creative telescoping principle [52].

2. Then we solve the derived recurrence in terms of the so-called d'Alembertian solutions [52]. Since this class covers the harmonic sums, we find all solutions in terms of harmonic sums.

3. Taking the initial values of the original input sum, we can combine the solutions found from step 2 in order to arrive at a closed form representation in terms of harmonic sums.

A detailed example for the sum (B.29) with all its computation steps has been carried out in [53]. In Appendix B we present the details for the calculation of a further example, Eq. (B.60), in Section B.8.

The results for new sums contributing are listed in Appendix B. In the calculation also more well-known sums are occurring which were found before in [7] or can be easily solved using the FORM-code [54] summer [16].

The $O(\varepsilon)$ contribution to $\hat{A}_{Q g}^{(2)}$, after mass renormalization, reads:

$$
\begin{aligned}
& \bar{a}_{Q g}^{(2)}(N) \\
& =T_{F} C_{F}\left\{\frac { N ^ { 2 } + N + 2 } { N ( N + 1 ) ( N + 2 ) } \left(16 S_{2,1,1}-8 S_{3,1}-8 S_{2,1} S_{1}+3 S_{4}-\frac{4}{3} S_{3} S_{1}-\frac{1}{2} S_{2}^{2}\right.\right. \\
& \left.-S_{2} S_{1}^{2}-\frac{1}{6} S_{1}^{4}+2 \zeta_{2} S_{2}-2 \zeta_{2} S_{1}^{2}-\frac{8}{3} \zeta_{3} S_{1}\right)-8 \frac{N^{2}-3 N-2}{N^{2}(N+1)(N+2)} S_{2,1} \\
& +\frac{2}{3} \frac{3 N+2}{N^{2}(N+2)} S_{1}^{3}+\frac{2}{3} \frac{3 N^{4}+48 N^{3}+43 N^{2}-22 N-8}{N^{2}(N+1)^{2}(N+2)} S_{3}+2 \frac{3 N+2}{N^{2}(N+2)} S_{2} S_{1} \\
& +4 \frac{S_{1}}{N^{2}} \zeta_{2}+\frac{2}{3} \frac{\left(N^{2}+N+2\right)\left(3 N^{2}+3 N+2\right)}{N^{2}(N+1)^{2}(N+2)} \zeta_{3}+\frac{P_{1}}{N^{3}(N+1)^{3}(N+2)} S_{2} \\
& +\frac{N^{4}-5 N^{3}-32 N^{2}-18 N-4}{N^{2}(N+1)^{2}(N+2)} S_{1}^{2} \\
& -2 \frac{2 N^{5}-2 N^{4}-11 N^{3}-19 N^{2}-44 N-12}{N^{2}(N+1)^{3}(N+2)} S_{1} \\
& \left.-\frac{5 N^{6}+15 N^{5}+36 N^{4}+51 N^{3}+25 N^{2}+8 N+4}{N^{3}(N+1)^{3}(N+2)} \zeta_{2}-\frac{P_{2}}{N^{5}(N+1)^{5}(N+2)}\right\} \\
& +T_{F} C_{A}\left\{\frac { N ^ { 2 } + N + 2 } { N ( N + 1 ) ( N + 2 ) } \left(16 S_{-2,1,1}-4 S_{2,1,1}-8 S_{-3,1}-8 S_{-2,2}-4 S_{3,1}\right.\right.
\end{aligned}
$$




$$
\begin{aligned}
& -\frac{2}{3} \beta^{\prime \prime \prime}+9 S_{4}-16 S_{-2,1} S_{1}+\frac{40}{3} S_{1} S_{3}+4 \beta^{\prime \prime} S_{1}-8 \beta^{\prime} S_{2}+\frac{1}{2} S_{2}^{2}-8 \beta^{\prime} S_{1}^{2} \\
+ & \left.5 S_{1}^{2} S_{2}+\frac{1}{6} S_{1}^{4}-\frac{10}{3} S_{1} \zeta_{3}-2 S_{2} \zeta_{2}-2 S_{1}^{2} \zeta_{2}-4 \beta^{\prime} \zeta_{2}-\frac{17}{5} \zeta_{2}^{2}\right) \\
+ & \frac{4\left(N^{2}-N-4\right)}{(N+1)^{2}(N+2)^{2}}\left(-4 S_{-2,1}+\beta^{\prime \prime}-4 \beta^{\prime} S_{1}\right) \\
- & \frac{16}{3} \frac{N^{5}+10 N^{4}+9 N^{3}+3 N^{2}+7 N+6}{(N-1) N^{2}(N+1)^{2}(N+2)^{2}} S_{3}+2 \frac{3 N^{3}-12 N^{2}-27 N-2}{N(N+1)^{2}(N+2)^{2}} S_{2} S_{1} \\
- & \frac{2}{3} \frac{N^{3}+8 N^{2}+11 N+2}{N(N+1)^{2}(N+2)^{2}} S_{1}^{3}-8 \frac{N^{2}+N-1}{(N+1)^{2}(N+2)^{2}} \zeta_{2} S_{1} \\
- & \frac{2}{3} \frac{9 N^{5}-10 N^{4}-11 N^{3}+68 N^{2}+24 N+16}{(N-1) N^{2}(N+1)^{2}(N+2)^{2}} \zeta_{3} \\
+ & 8 \frac{N^{4}+2 N^{3}+7 N^{2}+22 N+20}{(N+1)^{3}(N+2)^{3}} \beta^{\prime}-\frac{P_{3}}{(N-1) N^{3}(N+1)^{3}(N+2)^{3}} S_{2} \\
- & \frac{2 P_{4}}{(N-1) N^{3}(N+1)^{3}(N+2)^{2}} \zeta_{2}-\frac{P_{5}}{N(N+1)^{3}(N+2)^{3}} S_{1}^{2} \\
+ & \left.\frac{2 P_{6}}{N(N+1)^{4}(N+2)^{4}} S_{1}-\frac{2 P_{7}}{(N-1) N^{5}(N+1)^{5}(N+2)^{5}}\right\} .
\end{aligned}
$$

Here the argument $N$ of the harmonic sums, and $(N+1)$ in the function

$$
\begin{aligned}
& \beta(N)=\frac{1}{2}\left[\psi\left(\frac{N+1}{2}\right)-\psi\left(\frac{N}{2}\right)\right], \\
& S_{-1}(N)=(-1)^{N} \beta(N+1)-\ln (2)
\end{aligned}
$$

and in the polynomials $P_{i}(N)$ was omitted as well as the factor

$$
S_{\varepsilon}^{2} a_{s}^{2}\left(\frac{m^{2}}{\mu^{2}}\right)^{\varepsilon} .
$$

In the gluon and pure-singlet case we did not write the overall factor

$$
\frac{1+(-1)^{N}}{2} \text {. }
$$

It does not emerge generically in the non-singlet case. In accordance with the light-cone expansion only even integer moments contribute in the present case. The polynomials in Eq. (48) are

$$
\begin{aligned}
P_{1}= & 3 N^{6}+30 N^{5}+15 N^{4}-64 N^{3}-56 N^{2}-20 N-8, \\
P_{2}= & 24 N^{10}+136 N^{9}+395 N^{8}+704 N^{7}+739 N^{6}+407 N^{5}+87 N^{4}+27 N^{3} \\
& +45 N^{2}+24 N+4, \\
P_{3}= & N^{9}+21 N^{8}+85 N^{7}+105 N^{6}+42 N^{5}+290 N^{4}+600 N^{3}+456 N^{2} \\
& +256 N+64, \\
P_{4}= & \left(N^{3}+3 N^{2}+12 N+4\right)\left(N^{5}-N^{4}+5 N^{2}+N+2\right), \\
P_{5}= & N^{6}+6 N^{5}+7 N^{4}+4 N^{3}+18 N^{2}+16 N-8, \\
P_{6}= & 2 N^{8}+22 N^{7}+117 N^{6}+386 N^{5}+759 N^{4}+810 N^{3}+396 N^{2}+72 N+32,
\end{aligned}
$$




$$
\begin{aligned}
P_{7}= & 4 N^{15}+50 N^{14}+267 N^{13}+765 N^{12}+1183 N^{11}+682 N^{10}-826 N^{9}-1858 N^{8} \\
& -1116 N^{7}+457 N^{6}+1500 N^{5}+2268 N^{4}+2400 N^{3}+1392 N^{2} \\
& +448 N+64 .
\end{aligned}
$$

The flavor non-singlet and pure-singlet contributions read:

$$
\begin{aligned}
\bar{a}_{q q, Q}^{\mathrm{NS},(2)}= & T_{F} C_{F}\left\{\frac{4}{3} S_{4}+\frac{4}{3} S_{2} \zeta_{2}-\frac{8}{9} S_{1} \zeta_{3}-\frac{20}{9} S_{3}-\frac{20}{9} S_{1} \zeta_{2}+2 \frac{3 N^{2}+3 N+2}{9 N(N+1)} \zeta_{3}\right. \\
& +\frac{112}{27} S_{2}+\frac{3 N^{4}+6 N^{3}+47 N^{2}+20 N-12}{18 N^{2}(N+1)^{2}} \zeta_{2}-\frac{656}{81} S_{1} \\
& \left.+\frac{P_{8}}{648 N^{4}(N+1)^{4}}\right\} \\
P_{8}= & 1551 N^{8}+6204 N^{7}+15338 N^{6}+17868 N^{5}+8319 N^{4} \\
& +944 N^{3}+528 N^{2}-144 N-432 . \\
\bar{a}_{Q q}^{\mathrm{PS},(2)}= & T_{F} C_{F}\left\{-2 \frac{\left(5 N^{3}+7 N^{2}+4 N+4\right)\left(N^{2}+5 N+2\right)}{(N-1) N^{3}(N+1)^{3}(N+2)^{2}}\left(2 S_{2}+\zeta_{2}\right)\right. \\
& \left.\quad-\frac{4}{3} \frac{\left(N^{2}+N+2\right)^{2}}{(N-1) N^{2}(N+1)^{2}(N+2)}\left(3 S_{3}+\zeta_{3}\right)+2 \frac{P_{9}}{(N-1) N^{5}(N+1)^{5}(N+2)^{4}}\right\} \\
P_{9}= & 5 N^{11}+62 N^{10}+252 N^{9}+374 N^{8}-400 N^{6}+38 N^{7}-473 N^{5} \\
& -682 N^{4}-904 N^{3}-592 N^{2}-208 N-32 .
\end{aligned}
$$

The harmonic sums contributing to the individual diagrams, see Appendix A, are listed in Table 1.

Here we have already made use of the algebraic relations [55]. Moreover, two of the sums, $S_{-2,2}(N)$ and $S_{3,1}(N)$, can be related by structural relations [56] to other harmonic sums, i.e., they lie in corresponding equivalence classes and may be obtained by either rational argument relations and/or differentiation w.r.t. $N$. Reference to these equivalence classes is useful since the representation of these sums for $N \in \mathbf{C}$ needs not to be derived newly, except of differentiation which is easily carried out. Therefore the two-loop massive operator matrix elements to $O(\varepsilon)$ depend on six basic harmonic sums. In the complex Mellin- $N$ plane these functions, up to more simple terms due to the soft- and virtual corrections, are meromorphic functions with poles at the non-positive integers, which possess both an analytic regular asymptotic representation and recursion relations, through which they may be calculated. To apply the results obtained in the present calculation in Mellin space in a QCD-analysis of deeply inelastic structure functions, their scale evolution is first evaluated in Mellin space, incorporating the heavy flavor Wilson coefficients for complex values of $N$. This requires analytic continuations of the corresponding harmonic sums as worked out in [57].

The result in $x$-space is obtained by a single, fast numeric Mellin-inversion performed by a contour integral around the singularities of the problem located at the real axis left to some value $r$. In case one wants to include small- $x$ resummations as well, this can be done in a similar way, see [58].

We performed an independent check on our calculation evaluating fixed moments in $N$ for the un-renormalized diagrams using the Mellin-Barnes method $[59,60]$. Here we use an extension of a method developed for massless propagators in [61] to massive on-shell operator matrix elements [62]. The Mellin-Barnes integrals are evaluated numerically using the package MB [63]. 
Table 1

Complexity of the results in Mellin space for the individual diagrams in the unpolarized case, cf. [7], up to $O(\varepsilon)$

\begin{tabular}{|c|c|c|c|c|c|c|c|c|c|c|c|c|c|c|}
\hline Diagram & $S_{1}$ & $S_{2}$ & $S_{3}$ & $S_{4}$ & $S_{-2}$ & $S_{-3}$ & $S_{-4}$ & $S_{2,1}$ & $S_{-2,1}$ & $S_{-2,2}$ & $S_{3,1}$ & $S_{-3,1}$ & $S_{2,1,1}$ & $S_{-2,1,1}$ \\
\hline A & & + & + & & & & & & & & & & & \\
\hline B & + & + & + & + & & & & + & & & + & & + & \\
\hline $\mathrm{C}$ & & + & + & & & & & & & & & & & \\
\hline D & + & + & + & & & & & + & & & & & & \\
\hline E & + & + & + & & & & & + & & & & & & \\
\hline $\mathrm{F}$ & + & + & + & + & & & & + & & & & & + & \\
\hline G & + & + & + & & & & & + & & & & & & \\
\hline $\mathrm{H}$ & + & + & + & & & & & + & & & & & & \\
\hline I & + & + & + & + & + & + & + & + & + & + & + & + & + & + \\
\hline $\mathrm{J}$ & & + & + & & & & & & & & & & & \\
\hline K & & + & + & & & & & & & & & & & \\
\hline $\mathrm{L}$ & + & + & + & + & & & & + & & & + & & + & \\
\hline M & & + & + & & & & & & & & & & & \\
\hline $\mathrm{N}$ & + & + & + & + & + & + & + & + & + & + & + & + & + & + \\
\hline $\mathrm{O}$ & + & + & + & + & & & & + & & & + & & + & \\
\hline $\mathrm{P}$ & + & + & + & + & & & & + & & & + & & + & \\
\hline S & & + & + & & & & & & & & & & & \\
\hline $\mathrm{T}$ & & + & + & & & & & & & & & & & \\
\hline PS & & + & + & & & & & & & & & & & \\
\hline $\mathrm{NS}_{a}$ & & & & & & & & & & & & & & \\
\hline
\end{tabular}

In Table 2, we present the moments $N=2$ and $N=6$ for the more difficult two-loop diagrams, cf. [7], for the $O\left(1 / \varepsilon^{2}\right)$ to the $O\left(\varepsilon^{2}\right)$ terms.

A further test for the Abelian part of the first moment of the un-renormalized massive operator matrix element $A_{Q g}^{(2)}$ after mass renormalization, i.e., the term $\propto T_{F} C_{F}$, can be performed after analytic continuation of even values of $N$. This term is related to a corresponding contribution of the on-shell photon polarization function as noted in [6]. We apply this method to the $O(\varepsilon)$ term in Appendix C and find agreement.

\section{Conclusions}

We calculated the $O(\varepsilon)$ contributions to the massive operator matrix elements at $O\left(a_{s}^{2}\right)$ which contribute to the heavy flavor Wilson coefficients in deeply inelastic scattering to the non-powersuppressed contributions. In the renormalization of the heavy flavor Wilson coefficients to 3-loop order they contribute together with the single pole terms at $O\left(a_{s}\right)$. These terms, and the $O\left(a_{s}^{2} \varepsilon\right)$ contributions to the operator matrix element $A_{g g}(N)$ to be published soon, form all but the constant terms of the 3-loop heavy flavor unpolarized operator matrix elements needed to describe the 3-loop heavy flavor Wilson coefficients, together with the known 3-loop massless Wilson coefficients [11], in the region $Q^{2} \gg m^{2}$. In the calculation, we made use of the representation of the Feynman-parameter integrals in terms of generalized hypergeometric functions in a direct calculation, without applying the integration-by-parts method. The $\varepsilon$-expansion leads to new infinite sums which had to be solved by analytic and advanced algebraic methods. We checked our results for finite values of $N$, using the Mellin-Barnes method, and for a series of diagrams by a second program. Here, the calculation can be extended to higher corrections in $\varepsilon$. For $N=1$, one may compare in addition the terms $\propto T_{F} C_{F}$ in $\bar{a}_{Q g}^{(2)}$ with the corresponding contribution in the 
Table 2

Numerical values for the moments $N=2,6$ for the expansion of the un-renormalized matrix element $A_{Q g}^{2}$ for the terms $O\left(1 / \varepsilon^{2}\right)$ to $O\left(\varepsilon^{2}\right)$ for individual diagrams

\begin{tabular}{|c|c|c|c|c|c|}
\hline Diagram/order & $1 / \varepsilon^{2}$ & $1 / \varepsilon$ & 1 & $\varepsilon$ & $\varepsilon^{2}$ \\
\hline B $N=2$ & -8 & 4.66666 & -8.82690 & 2.47728 & -5.69523 \\
\hline$N=6$ & -7.73333 & 0.81936 & -8.89777 & -1.84111 & -7.25674 \\
\hline $\mathrm{C} N=2$ & -4 & 19.8 & -3.61715 & 17.33108 & 3.26445 \\
\hline$N=6$ & -1.33333 & 8.26984 & -1.34024 & 7.12612 & 1.38782 \\
\hline $\mathrm{D} N=2$ & -8 & 7.86666 & -6.34542 & 4.71236 & -2.18586 \\
\hline$N=6$ & -2.66666 & -0.69523 & -2.60657 & -1.74990 & -2.37611 \\
\hline $\mathrm{E} N=2$ & 8.88889 & -11.2593 & 9.82824 & -12.8921 & 2.39145 \\
\hline$N=6$ & 2.93878 & -4.24257 & 3.39094 & -4.3892 & 0.826978 \\
\hline $\mathrm{F} N=2$ & 2.66667 & -4.88889 & 9.1707 & -1.2618 & 8.10105 \\
\hline$N=6$ & 1.65714 & -3.43645 & 6.12836 & -0.81895 & 5.43478 \\
\hline $\mathrm{G} N=2$ & 2.66666 & -9.55555 & 4.59662 & -8.92015 & 1.07313 \\
\hline$N=6$ & 0.57142 & -2.00204 & 1.04814 & -1.89142 & 0.32219 \\
\hline $\mathrm{H} N=2$ & -2.66666 & 3.55555 & -3.76328 & 4.57782 & -2.02934 \\
\hline$N=6$ & -0.57143 & 0.83061 & -0.9478 & 1.08896 & -0.55443 \\
\hline I $N=2$ & 0 & 0 & 0 & 0 & 0 \\
\hline$N=6$ & $\begin{array}{c}0.34285 C_{A} \\
+0 . C_{F}\end{array}$ & $\begin{array}{l}-0.38979 C_{A} \\
\quad+0.30793 C_{F}\end{array}$ & $\begin{array}{l}0.45862 C_{A} \\
\quad-0.00949 C_{F}\end{array}$ & $\begin{array}{l}-0.33621 C_{A} \\
\quad+0.32243 C_{F}\end{array}$ & $\begin{array}{l}0.27344 C_{A} \\
\quad+0.10405 C_{F}\end{array}$ \\
\hline $\mathrm{L} N=2$ & 22.22222 & -8.07407 & 21.63853 & -1.09400 & 14.38017 \\
\hline$N=6$ & 9.42675 & 0.69179 & 9.99619 & 4.12879 & 8.49503 \\
\hline $\mathrm{M} N=2$ & -0.44444 & -1.66666 & -0.25375 & -2.19775 & -0.72592 \\
\hline$N=6$ & 0.09977 & -0.21941 & 0.12862 & -0.23387 & 0.03250 \\
\hline $\mathrm{N} N=2$ & 5.77778 & -1.70370 & 4.24641 & -0.06679 & 2.27892 \\
\hline$N=6$ & 4.26462 & 0.17238 & 4.61023 & 1.71768 & 3.87767 \\
\hline $\mathrm{O} N=2$ & -4.44444 & 3.14815 & -4.96659 & 2.01274 & -3.0498 \\
\hline$N=6$ & -1.03605 & 0.56098 & -1.12332 & 0.27406 & -0.73034 \\
\hline
\end{tabular}

2-loop on-shell photon propagator. The terms $\bar{a}^{(2)}(N)$ can be expressed in terms of polynomials of the basic nested harmonic sums up to weight $w=4$ and derivatives thereof. They belong to the complexity-class of the general two-loop Wilson coefficients or hard scattering cross sections in massless QED and QCD found for space- and time-like unpolarized and polarized anomalous dimensions, massless Wilson coefficients for deeply inelastic scattering, parton fragmentation, the Drell-Yan process, hadronic Higgs- and pseudoscalar Higgs production in the heavy mass limit as well as the soft- and virtual contribution to Bhabha-scattering, cf. [65], and are described by six basic functions and their derivatives in Mellin space. Their analytic continuation to complex values of $N$ is known in explicit form. The package Sigma [19] proved to be a useful tool to solve the sums occurring in the present problem and was extended accordingly. One advantage to seek for solutions of the recurrences emerging in $\Pi \Sigma$-fields consists in finding irreducible structures for the representation. In the present calculation these were nested harmonic sums. In even more complicated single scale problems in higher orders, this needs not to be the case. The new basis elements, however, would be uniquely found applying the present procedure.

\section{Acknowledgements}

We would like to thank W. Bernreuther, K. Chetyrkin, F. Jegerlehner, and M. Steinhauser for useful conversations. We thank P. Paule for his interest in this work. This work was supported in part by DFG Sonderforschungsbereich Transregio 9, Computergestützte Theoretische 
Teilchenphysik, Spezialforschungsbereich F1305, project P20347-N18 of the Austrian FWF, and Studienstiftung des Deutschen Volkes. We used the code axodraw [67] to draw Feynman diagrams.

\section{Appendix A. The $O(\varepsilon)$ terms for the individual diagrams}

In the following we list the results for the individual diagrams for comparisons and to illustrate the analytic structures emerging in the calculation. The calculation is performed in Feynman gauge. Again we suppress the factor (51) and the argument $N$ in the sums and polynomials.

$$
\begin{aligned}
& \bar{A}_{a}^{Q g}=T_{F} C_{F}\left\{4 \frac{3 S_{3}+\zeta_{3}}{3 N^{2}(N+1)}+2 \frac{2 N^{3}-N-2}{N^{3}(N+1)^{2}(N+2)}\left(2 S_{2}+\zeta_{2}\right)\right. \\
& \left.-\frac{2 \hat{P}_{1}}{N^{5}(N+1)^{4}(N+2)^{3}}\right\} \\
& \hat{P}_{1}=2 N^{9}-16 N^{8}-89 N^{7}-166 N^{6}-135 N^{5}-6 N^{4}+85 N^{3}+94 N^{2}+44 N+8 \text {. } \\
& \bar{A}_{b}^{Q g}=T_{F} C_{F}\left\{\frac { 1 } { N } \left(8 S_{2,1,1}-8 S_{3,1}+11 S_{4}-8 S_{2,1} S_{1}-\frac{4}{3} S_{3} S_{1}\right.\right. \\
& \left.+\frac{7}{2} S_{2}^{2}-S_{2} S_{1}^{2}-\frac{1}{6} S_{1}^{4}+6 S_{2} \zeta_{2}-2 S_{1}^{2} \zeta_{2}-\frac{8}{3} S_{1} \zeta_{3}+\frac{8}{3} \zeta_{3}\right) \\
& +\frac{N^{2}+7 N+2}{N(N+1)(N+2)}\left(8 S_{2,1}+2 S_{2} S_{1}+\frac{2}{3} S_{1}^{3}+4 S_{1} \zeta_{2}\right) \\
& -4 \frac{N^{5}+3 N^{4}+19 N^{3}+37 N^{2}+16 N+4}{N^{2}(N+1)^{2}(N+2)^{2}} S_{2}-4 \frac{N^{3}+9 N^{2}+8 N+4}{N^{2}(N+2)^{2}} S_{1}^{2} \\
& -8 \frac{N^{2}+5 N+2}{N(N+1)(N+2)} \zeta_{2}+\frac{4}{3} \frac{N^{2}-17 N+2}{N(N+1)(N+2)} S_{3}+\frac{16 \hat{P}_{2}}{N^{2}(N+1)^{3}(N+2)^{3}} S_{1} \\
& \left.-\frac{16 \hat{P}_{3}}{N(N+1)^{4}(N+2)^{3}}\right\} \\
& \hat{P}_{2}=N^{7}+14 N^{6}+65 N^{5}+153 N^{4}+197 N^{3}+134 N^{2}+44 N+8 \text {, } \\
& \hat{P}_{3}=2 N^{7}+27 N^{6}+130 N^{5}+306 N^{4}+385 N^{3}+266 N^{2}+100 N+16 . \\
& \bar{A}_{c}^{Q g}=T_{F} C_{F}\left\{\frac{2}{N}\left(5 S_{3}-\frac{1}{3} \zeta_{3}\right)-2 \frac{7 N^{3}+29 N^{2}+15 N+2}{N^{2}(N+1)(N+2)} S_{2}\right. \\
& \left.+\frac{13 N^{4}+82 N^{3}+82 N^{2}+N-6}{N^{2}(N+1)(N+2)(N+3)} \zeta_{2}+\frac{\hat{P}_{4}}{N^{4}(N+1)^{3}(N+2)^{3}(N+3)}\right\}, \\
& \hat{P}_{4}=32 N^{10}+448 N^{9}+2177 N^{8}+5123 N^{7}+6312 N^{6}+3863 N^{5}+902 N^{4} \\
& +9 N^{3}-74 N^{2}-68 N-24 . \\
& \bar{A}_{d}^{Q g}=T_{F} C_{F}\left\{\frac{1}{N}\left(-4 S_{2,1}-\frac{2}{3} S_{3}-S_{2} S_{1}-\frac{1}{3} S_{1}^{3}-2 S_{1} \zeta_{2}-\frac{4}{3} \zeta_{3}\right)\right. \\
& +\frac{N^{4}+8 N^{3}+43 N^{2}+36 N+12}{N^{2}(N+1)^{2}(N+2)}\left(S_{2}+S_{1}^{2}\right)+2 \frac{N^{3}+10 N^{2}+59 N+42}{N(N+1)(N+2)(N+3)} \zeta_{2} \\
& \left.-\frac{4 \hat{P}_{5}}{N^{2}(N+1)^{3}(N+2)^{2}} S_{1}+\frac{4 \hat{P}_{6}}{N(N+1)^{4}(N+2)^{3}(N+3)}\right\} \text {, }
\end{aligned}
$$




$$
\begin{aligned}
& \hat{P}_{5}=N^{6}+8 N^{5}+79 N^{4}+207 N^{3}+205 N^{2}+96 N+20 \text {, } \\
& \hat{P}_{6}=2 N^{8}+24 N^{7}+262 N^{6}+1371 N^{5}+3514 N^{4}+4775 N^{3}+3544 N^{2}+1404 N+240 . \\
& \bar{A}_{e}^{Q g}=T_{F}\left[C_{F}-\frac{C_{A}}{2}\right]\left\{-2 \frac{N+2}{N(N+1)}\left(2 S_{2,1}+S_{1} \zeta_{2}\right)\right. \\
& -\frac{2}{3} \frac{13 N^{4}+60 N^{3}+111 N^{2}+4 N-36}{N(N+1)^{2}(N+2)(N+3)} S_{3} \\
& -\frac{1}{3} \frac{N^{3}-N^{2}-8 N-36}{N(N+1)(N+2)(N+3)}\left(3 S_{2} S_{1}+S_{1}^{3}\right)+\frac{4}{3} \frac{N+3}{(N+1)^{2}} \zeta_{3} \\
& -2 \frac{3 N^{3}+9 N^{2}+12 N+4}{N(N+1)^{3}(N+2)} \zeta_{2}+\frac{\hat{P}_{7}}{N^{2}(N+1)^{3}(N+2)(N+3)} S_{2} \\
& +\frac{4 N^{5}+11 N^{4}+15 N^{3}-86 N^{2}-92 N-24}{N^{2}(N+1)^{2}(N+2)(N+3)} S_{1}^{2} \\
& \left.-2 \frac{\hat{P}_{8}}{N^{2}(N+1)^{3}(N+2)^{2}(N+3)} S_{1}-2 \frac{\hat{P}_{9}}{N^{3}(N+1)^{5}(N+2)^{3}(N+3)}\right\}, \\
& \hat{P}_{7}=20 N^{6}+119 N^{5}+290 N^{4}+105 N^{3}-290 N^{2}-212 N-24 \text {, } \\
& \hat{P}_{8}=8 N^{7}+62 N^{6}+181 N^{5}+127 N^{4}-226 N^{3}-404 N^{2}-296 N-96 \text {, } \\
& \hat{P}_{9}=38 N^{10}+394 N^{9}+1775 N^{8}+4358 N^{7}+6323 N^{6}+5788 N^{5}+3626 N^{4}+1462 N^{3} \\
& +100 N^{2}-184 N-48 \text {. } \\
& \bar{A}_{f}^{Q g}=T_{F}\left[C_{F}-\frac{C_{A}}{2}\right]\left\{\frac{4}{N}\left(2 S_{2,1,1}-2 S_{4}-S_{2}^{2}-\zeta_{2} S_{2}\right)+\frac{16\left(S_{1}-1\right) \zeta_{3}}{3(N+1)(N+2)}\right. \\
& -8 \frac{2 N^{2}-5 N-2}{N^{2}(N+1)(N+2)} S_{2,1}+\frac{4}{3} \frac{26 N^{2}-33 N-10}{N^{2}(N+1)(N+2)} S_{3} \\
& +\frac{2}{3} \frac{2 N^{2}-3 N+2}{N^{2}(N+1)(N+2)}\left(3 S_{2} S_{1}+S_{1}^{3}\right)+4 \frac{5 N+2}{N^{2}(N+1)(N+2)} S_{1} \zeta_{2} \\
& -2 \frac{2 N^{3}+15 N^{2}+12 N-4}{N(N+1)^{2}(N+2)^{2}} S_{1}^{2}+2 \frac{14 N^{3}+85 N^{2}+132 N+52}{N(N+1)^{2}(N+2)^{2}} S_{2} \\
& +4 \frac{2 N^{5}+18 N^{4}+25 N^{3}+10 N^{2}+44 N+56}{N(N+1)^{3}(N+2)^{3}} S_{1}-\frac{8 \zeta_{2}}{(N+1)^{2}(N+2)} \\
& \left.-8 \frac{6 N^{5}+52 N^{4}+178 N^{3}+309 N^{2}+264 N+84}{(N+1)^{4}(N+2)^{3}}\right\} \text {. } \\
& \bar{A}_{g}^{Q g}=T_{F} C_{F}\left\{\frac{12 S_{2,1}-58 S_{3}+3 S_{2} S_{1}+S_{1}^{3}+6 \zeta_{2} S_{1}+8 \zeta_{3}}{3(N+1)(N+2)}\right. \\
& +\frac{(9 N+8)\left(5 N^{2}+9 N-1\right)}{N(N+1)^{2}(N+2)^{2}} S_{2}-\frac{3 N^{3}+31 N^{2}+45 N+8}{N(N+1)^{2}(N+2)^{2}} S_{1}^{2} \\
& -2 \frac{17 N^{2}+47 N+28}{(N+1)^{2}(N+2)^{2}} \zeta_{2}+2 \frac{6 N^{5}+104 N^{4}+376 N^{3}+514 N^{2}+277 N+48}{N(N+1)^{3}(N+2)^{3}} S_{1} \\
& \left.-\frac{2 \hat{P}_{10}}{(N+1)^{4}(N+2)^{4}}\right\} \text {, }
\end{aligned}
$$




$$
\begin{aligned}
& \hat{P}_{10}=74 N^{6}+722 N^{5}+2697 N^{4}+4960 N^{3}+4700 N^{2}+2143 N+368 . \\
& \bar{A}_{h}^{Q g}=T_{F}\left[C_{F}-\frac{C_{A}}{2}\right]\left\{\frac{4(N+3)}{N(N+1)(N+2)}\left(2 S_{2,1}+S_{1} \zeta_{2}\right)\right. \\
& -\frac{4}{3} \frac{N^{2}-26 N+9}{N(N+1)(N+2)(N+3)} S_{3}-\frac{2}{3} \frac{N^{2}-2 N+9}{N(N+1)(N+2)(N+3)}\left(3 S_{2} S_{1}+S_{1}^{3}\right) \\
& -\frac{8 \zeta_{3}}{3(N+1)(N+2)}-2 \frac{N^{2}+7 N+8}{(N+1)^{2}(N+2)^{2}} \zeta_{2} \\
& -\frac{N^{4}+115 N^{3}+335 N^{2}+265 N+84}{N(N+1)^{2}(N+2)^{2}(N+3)} S_{2}+\frac{7 N^{4}+N^{3}-31 N^{2}-37 N-36}{N(N+1)^{2}(N+2)^{2}(N+3)} S_{1}^{2} \\
& \left.-\frac{2 \hat{P}_{11}}{N(N+1)^{3}(N+2)^{3}(N+3)} S_{1}+\frac{2 \hat{P}_{12}}{(N+1)^{4}(N+2)^{4}(N+3)}\right\} \text {, } \\
& \hat{P}_{11}=10 N^{6}+38 N^{5}-43 N^{4}-533 N^{3}-1267 N^{2}-1269 N-456 \text {, } \\
& \hat{P}_{12}=36 N^{7}+344 N^{6}+1287 N^{5}+2143 N^{4}+818 N^{3}-2153 N^{2}-2795 N-960 . \\
& \bar{A}_{i}^{Q g}=T_{F} C_{A}\left\{\frac { 8 } { N + 2 } \left(4 S_{-2,1,1}-2 S_{-3,1}-2 S_{-2,2}-4 S_{-2,1} S_{1}+S_{-4}+2 S_{-3} S_{1}\right.\right. \\
& \left.+2 S_{-2} S_{2}+2 S_{-2} S_{1}^{2}+S_{-2} \zeta_{2}\right)+8 \frac{N^{2}-N-4}{(N+1)(N+2)^{2}}\left(2 S_{-2,1}-S_{-3}-2 S_{-2} S_{1}\right) \\
& +\frac{1}{(N+1)(N+2)}\left(4(4 N+7) S_{2,1,1}-4(6 N+7) S_{3,1}-4(4 N+5) S_{2,1} S_{1}\right. \\
& +\frac{32 N+27}{2} S_{4}+\frac{2}{3}(36 N+35) S_{3} S_{1}-\frac{16 N+25}{4} S_{2}^{2}+\frac{16 N+15}{2} S_{2} S_{1}^{2}-\frac{1}{12} S_{1}^{4} \\
& \left.-S_{1}^{2} \zeta_{2}+\frac{4}{3} S_{1} \zeta_{3}+(4 N+3) S_{2} \zeta_{2}\right)+2 \frac{N^{3}+9 N^{2}+17 N+8}{N(N+1)^{2}(N+2)^{2}} S_{1} \zeta_{2} \\
& +4 \frac{2 N^{4}+N^{3}-N^{2}+9 N+8}{N(N+1)^{2}(N+2)^{2}} S_{2,1}-\frac{2}{3} \frac{18 N^{4}+3 N^{3}-67 N^{2}-39 N+8}{N(N+1)^{2}(N+2)^{2}} S_{3} \\
& -\frac{8 N^{4}-3 N^{3}-47 N^{2}-29 N+8}{N(N+1)^{2}(N+2)^{2}} S_{2} S_{1}+\frac{1}{3} \frac{3 N^{3}+7 N^{2}-3 N-8}{N(N+1)^{2}(N+2)^{2}} S_{1}^{3} \\
& -\frac{4(N+4) \zeta_{3}}{3(N+1)(N+2)^{2}}+8 \frac{N^{4}+2 N^{3}+7 N^{2}+22 N+20}{(N+1)^{2}(N+2)^{3}} S_{-2} \\
& +\frac{\hat{P}_{13} S_{2}}{N(N+1)^{3}(N+2)^{3}}-\frac{6 N^{5}+40 N^{4}+92 N^{3}+94 N^{2}+49 N+16}{N(N+1)^{3}(N+2)^{3}} S_{1}^{2} \\
& \left.-2 \frac{2 N^{3}+8 N^{2}+19 N+16}{(N+1)^{2}(N+2)^{3}} \zeta_{2}+\frac{2 \hat{P}_{14}}{N(N+1)^{4}(N+2)^{4}} S_{1}-\frac{2 \hat{P}_{15}}{(N+1)^{4}(N+2)^{5}}\right\} \\
& +T_{F} C_{F}\left\{\frac { 1 } { ( N + 1 ) ( N + 2 ) } \left(-32 S_{2,1,1}+16 S_{3,1}-6 S_{4}+\frac{8}{3} S_{3} S_{1}+16 S_{2,1} S_{1}\right.\right. \\
& \left.+S_{2}^{2}+2 S_{2} S_{1}^{2}+\frac{1}{3} S_{1}^{4}-4 S_{2} \zeta_{2}+4 S_{1}^{2} \zeta_{2}+32 \zeta_{2}+224\right)-16 \frac{2 S_{2,1}+S_{1} \zeta_{2}}{N(N+2)} \\
& -\frac{4}{3} \frac{3 N-2}{N(N+1)(N+2)}\left(3 S_{2} S_{1}+S_{1}^{3}\right)+\frac{16}{3} \frac{3 N+1}{N(N+1)(N+2)} S_{3}
\end{aligned}
$$




$$
\begin{aligned}
& -8 \frac{2 N^{2}+2 N-1}{N(N+1)^{2}(N+2)} S_{2}+8 \frac{3 N^{2}+3 N+1}{N(N+1)^{2}(N+2)} S_{1}^{2} \\
& \left.-8 \frac{11 N^{3}+33 N^{2}+38 N+14}{N(N+1)^{3}(N+2)} S_{1}\right\} \\
& \hat{P}_{13}=4 N^{6}+10 N^{5}+12 N^{4}+8 N^{3}-18 N^{2}-37 N-16 \text {, } \\
& \hat{P}_{14}=10 N^{7}+114 N^{6}+533 N^{5}+1374 N^{4}+2144 N^{3}+2027 N^{2}+1057 N+224, \\
& \hat{P}_{15}=20 N^{7}+236 N^{6}+1202 N^{5}+3384 N^{4}+5688 N^{3}+5720 N^{2}+3195 N+768 . \\
& \bar{A}_{j}^{Q g}=T_{F} C_{A}\left\{-\frac{2}{3} \frac{4 N^{2}+4 N-5}{N^{2}(N+1)^{2}}\left(3 S_{3}+\zeta_{3}\right)+\frac{\hat{P}_{16}}{N^{5}(N+1)^{5}(N+2)^{3}}\right. \\
& \left.+\frac{4 N^{5}+22 N^{4}+11 N^{3}+13 N^{2}+35 N+10}{N^{3}(N+1)^{3}(N+2)}\left(2 S_{2}+\zeta_{2}\right)\right\}, \\
& \hat{P}_{16}=28 N^{10}+148 N^{9}+342 N^{8}+285 N^{7}-212 N^{6}-114 N^{5}+1117 N^{4} \\
& +1587 N^{3}+826 N^{2}+260 N+40 . \\
& \bar{A}_{k}^{Q g}=T_{F} C_{A}\left\{\frac{2}{3} \frac{3 N^{2}-23 N-20}{(N-1) N(N+1)^{2}(N+2)}\left(3 S_{3}+\zeta_{3}\right)-\frac{\hat{P}_{17}}{(N-1) N(N+1)^{5}(N+2)^{4}}\right. \\
& \left.-\frac{10 N^{4}+7 N^{3}+51 N^{2}+172 N+112}{(N-1) N(N+1)^{3}(N+2)^{2}}\left(2 S_{2}+\zeta_{2}\right)\right\} \\
& \hat{P}_{17}=14 N^{8}+70 N^{7}+96 N^{6}-375 N^{5}-1493 N^{4}-1056 N^{3}+2392 N^{2} \\
& +4192 N+1792 \text {. } \\
& \bar{A}_{l}^{Q g}=T_{F} C_{A}\left\{\frac { 1 } { N } \left(-4 S_{2,1,1}+4 S_{3,1}+\frac{5}{2} S_{4}+4 S_{2,1} S_{1}+\frac{2}{3} S_{3} S_{1}+\frac{9}{4} S_{2}^{2}+\frac{1}{2} S_{2} S_{1}^{2}+S_{2} \zeta_{2}\right.\right. \\
& \left.+\frac{1}{12} S_{1}^{4}+S_{1}^{2} \zeta_{2}+\frac{4}{3} S_{1} \zeta_{3}\right)+\frac{2}{N(N+1)}\left(-4 S_{2,1}-S_{2} S_{1}-\frac{1}{3} S_{1}^{3}-2 S_{1} \zeta_{2}\right) \\
& -\frac{2}{3} \frac{6 N^{3}+5 N^{2}-4 N-6}{N^{2}(N+1)^{2}} S_{3}+\frac{8 N^{5}+18 N^{4}+11 N^{3}+N^{2}+6 N+4}{N^{3}(N+1)^{3}} S_{2} \\
& -\frac{(N+2)(2 N+1)}{N^{2}(N+1)^{2}} S_{1}^{2}+2 \frac{7 N^{3}+15 N^{2}+7 N+4}{N^{2}(N+1)^{3}} S_{1} \\
& +\frac{2}{3} \frac{2 N^{3}+5 N^{2}+4 N+2}{N^{2}(N+1)^{2}} \zeta_{3}-\frac{\hat{P}_{18} \zeta_{2}}{N^{3}(N+1)^{3}(N+2)} \\
& \left.-\frac{\hat{P}_{19}}{N^{5}(N+1)^{5}(N+2)}\right\} \text {, } \\
& \hat{P}_{18}=4 N^{6}+30 N^{5}+55 N^{4}+38 N^{3}+4 N^{2}-10 N-4 \text {, } \\
& \hat{P}_{19}=16 N^{10}+152 N^{9}+454 N^{8}+628 N^{7}+447 N^{6}+180 N^{5}+52 N^{4} \\
& -10 N^{3}-28 N^{2}-18 N-4 \text {, } \\
& \bar{A}_{m}^{Q g}=T_{F} C_{A}\left\{\frac{2}{3} \frac{N^{2}-2 N-2}{N^{2}(N+1)^{2}}\left(3 S_{3}+\zeta_{3}\right)\right. \\
& -\frac{2 N^{5}+11 N^{4}+12 N^{3}+2 N^{2}+6 N+4}{N^{3}(N+1)^{3}(N+2)}\left(2 S_{2}+\zeta_{2}\right)
\end{aligned}
$$




$$
\begin{aligned}
& \left.-\frac{6 N^{8}+28 N^{7}+53 N^{6}+30 N^{5}-14 N^{4}+2 N^{3}+18 N^{2}+14 N+4}{N^{5}(N+1)^{5}(N+2)}\right\}, \\
& \bar{A}_{n}^{Q g}=T_{F} C_{A}\left\{\frac { 4 ( N - 1 ) } { N ( N + 1 ) } \left(-4 S_{-2,1,1}+2 S_{-3,1}+2 S_{-2,2}-S_{-4}+4 S_{-2,1} S_{1}-2 S_{-3} S_{1}\right.\right. \\
& \left.-2 S_{-2} S_{2}-2 S_{-2} S_{1}^{2}-S_{-2} \zeta_{2}\right)+\frac{2 N^{2}+3 N+2}{24 N(N+1)(N+2)}\left(S_{1}^{4}+12 S_{1}^{2} \zeta_{2}+16 \zeta_{3} S_{1}\right) \\
& -2 \frac{6 N^{2}+7 N-6}{N(N+1)(N+2)}\left(S_{2,1,1}-S_{2,1} S_{1}\right)+2 \frac{8 N^{2}+9 N-10}{N(N+1)(N+2)} S_{3,1} \\
& -\frac{54 N^{2}+97 N-10}{4 N(N+1)(N+2)} S_{4}-\frac{34 N^{2}+33 N-74}{3 N(N+1)(N+2)} S_{3} S_{1}+\frac{2 N^{2}-37 N-78}{8 N(N+1)(N+2)} S_{2}^{2} \\
& -\frac{14 N^{2}+13 N-34}{4 N(N+1)(N+2)} S_{2} S_{1}^{2}-\frac{10 N^{2}+21 N+6}{2 N(N+1)(N+2)} S_{2} \zeta_{2}+8 \frac{N^{2}-N-4}{(N+1)^{2}(N+2)} \\
& \times\left(-2 S_{-2,1}+S_{-3}+2 S_{-2} S_{1}\right) \\
& -2 \frac{5 N^{5}+10 N^{4}-20 N^{3}-62 N^{2}-40 N-8}{N^{2}(N+1)^{2}(N+2)^{2}} S_{2,1}-\frac{2 N(N+3)}{3(N+1)^{2}(N+2)} \zeta_{3} \\
& +\frac{35 N^{5}+66 N^{4}-118 N^{3}-294 N^{2}-152 N-40}{3 N^{2}(N+1)^{2}(N+2)^{2}} S_{3} \\
& -\frac{N^{5}+6 N^{4}+4 N^{3}-30 N^{2}-40 N-8}{N^{2}(N+1)^{2}(N+2)^{2}} \zeta_{2} S_{1} \\
& +\frac{15 N^{5}+10 N^{4}-100 N^{3}-98 N^{2}+40 N+8}{2 N^{2}(N+1)^{2}(N+2)^{2}} S_{2} S_{1} \\
& -\frac{N^{5}+6 N^{4}+4 N^{3}-30 N^{2}-40 N-8}{6 N^{2}(N+1)^{2}(N+2)^{2}} S_{1}^{3}-8 \frac{N^{4}+2 N^{3}+7 N^{2}+22 N+20}{(N+1)^{3}(N+2)^{2}} S_{-2} \\
& -\frac{\hat{P}_{20} S_{2}-\hat{P}_{21} S_{1}^{2}}{2 N(N+1)^{3}(N+2)^{3}}+\frac{2 N^{4}+11 N^{3}+15 N^{2}+12 N+8}{(N+1)^{3}(N+2)^{2}} \zeta_{2} \\
& \left.-\frac{\hat{P}_{22} S_{1}}{N(N+1)^{4}(N+2)^{4}}+\frac{\hat{P}_{23}}{(N+1)^{5}(N+2)^{4}}\right\} \text {, } \\
& \hat{P}_{20}=6 N^{6}+36 N^{5}+60 N^{4}-99 N^{3}-390 N^{2}-316 N-40 \text {, } \\
& \hat{P}_{21}=2 N^{6}+20 N^{5}+40 N^{4}-45 N^{3}-170 N^{2}-100 N+8 \text {, } \\
& \hat{P}_{22}=4 N^{8}+50 N^{7}+224 N^{6}+544 N^{5}+927 N^{4}+1140 N^{3}+712 N^{2}-64 N-208 \text {, } \\
& \hat{P}_{23}=8 N^{8}+86 N^{7}+370 N^{6}+805 N^{5}+807 N^{4}-16 N^{3}-772 N^{2}-568 N-96 . \\
& \bar{A}_{o}^{Q g}=T_{F} C_{A}\left\{\frac { 1 } { 2 N ( N + 2 ) } \left(8 S_{2,1,1}-8 S_{3,1}-5 S_{4}-8 S_{1} S_{2,1}-\frac{4}{3} S_{3} S_{1}-\frac{9}{2} S_{2}^{2}-S_{2} S_{1}^{2}\right.\right. \\
& \left.-\frac{1}{6} S_{1}^{4}-2 S_{2} \zeta_{2}-2 S_{1}^{2} \zeta_{2}-\frac{8}{3} S_{1} \zeta_{3}\right)+\frac{2 N^{2}+9 N+12}{2 N(N+1)(N+2)^{2}}\left(4 S_{2,1}+S_{2} S_{1}\right. \\
& \left.+\frac{1}{3} S_{1}^{3}+2 S_{1} \zeta_{2}\right)-\frac{2}{3} \frac{N^{2}+7 N+8}{(N+1)^{2}(N+2)^{2}} \zeta_{3}+\frac{1}{3} \frac{14 N^{3}+41 N^{2}+51 N+36}{N(N+1)^{2}(N+2)^{2}} S_{3} \\
& -\frac{12 N^{5}+124 N^{4}+472 N^{3}+817 N^{2}+641 N+176}{2 N(N+1)^{3}(N+2)^{3}} S_{2}
\end{aligned}
$$




$$
\begin{aligned}
& +\frac{4 N^{4}+16 N^{3}-4 N^{2}-61 N-48}{2 N(N+1)^{2}(N+2)^{3}} S_{1}^{2}+\frac{N\left(11 N^{3}+56 N^{2}+92 N+49\right)}{(N+1)^{3}(N+2)^{3}} \zeta_{2} \\
& \left.-\frac{\hat{P}_{24} S_{1}}{N(N+1)^{3}(N+2)^{4}}+\frac{\hat{P}_{25}}{(N+1)^{5}(N+2)^{5}}\right\} \\
& \hat{P}_{24}=16 N^{6}+101 N^{5}+194 N^{4}+4 N^{3}-421 N^{2}-501 N-192 \text {, } \\
& \hat{P}_{25}=62 N^{8}+668 N^{7}+3073 N^{6}+7849 N^{5}+12052 N^{4}+11127 N^{3} \\
& +5640 N^{2}+1065 N-128 \text {. } \\
& \bar{A}_{p}^{Q g}=T_{F} C_{A}\left\{\frac { N - 4 } { 2 N ( N + 1 ) ( N + 2 ) } \left(4 S_{2,1,1}-4 S_{3,1}-4 S_{2,1} S_{1}-\frac{2}{3} S_{3} S_{1}-\frac{1}{2} S_{2} S_{1}^{2}-\frac{1}{12} S_{1}^{4}\right.\right. \\
& \left.-S_{1}^{2} \zeta_{2}-\frac{4}{3} S_{1} \zeta_{3}\right)+\frac{N^{3}-17 N^{2}-41 N-16}{2 N(N+1)^{2}(N+2)^{2}}\left(4 S_{2,1}+S_{2} S_{1}+\frac{1}{3} S_{1}^{3}+2 S_{1} \zeta_{2}\right) \\
& +\frac{11 N+20}{4 N(N+1)(N+2)} S_{4}+\frac{7 N+36}{8 N(N+1)(N+2)} S_{2}^{2}+\frac{3 N+4}{2 N(N+1)(N+2)} S_{2} \zeta_{2} \\
& -\frac{1}{3} \frac{11 N^{3}+17 N^{2}-N+16}{N(N+1)^{2}(N+2)^{2}} S_{3}-\frac{2}{3} \frac{N+4}{(N+1)(N+2)^{2}} \zeta_{3} \\
& +\frac{10 N^{5}+48 N^{4}+122 N^{3}+222 N^{2}+213 N+64}{2 N(N+1)^{3}(N+2)^{3}} S_{2} \\
& +\frac{2 N^{5}+48 N^{4}+174 N^{3}+242 N^{2}+161 N+64}{2 N(N+1)^{3}(N+2)^{3}} S_{1}^{2} \\
& +\frac{4 N^{3}+26 N^{2}+51 N+32}{(N+1)^{2}(N+2)^{3}} \zeta_{2}-\frac{\hat{P}_{26} S_{1}}{N(N+1)^{4}(N+2)^{4}} \\
& \left.+\frac{\hat{P}_{27}}{(N+1)^{4}(N+2)^{5}}\right\} \\
& \hat{P}_{26}=8 N^{7}+120 N^{6}+595 N^{5}+1538 N^{4}+2432 N^{3}+2419 N^{2}+1329 N+256 \text {, } \\
& \hat{P}_{27}=22 N^{7}+266 N^{6}+1360 N^{5}+3826 N^{4}+6400 N^{3}+6376 N^{2}+3515 N+832 . \\
& \bar{A}_{S}^{Q g}=T_{F} C_{A}\left\{-\frac{2}{3} \frac{3 S_{3}+\zeta_{3}}{N^{2}(N+1)^{2}}+\frac{2 N^{3}+N^{2}-3 N-1}{N^{3}(N+1)^{3}}\left(2 S_{2}+\zeta_{2}\right)\right. \\
& \left.+\frac{\hat{P}_{28}}{N^{5}(N+1)^{5}(N+2)^{2}}\right\} \text {, } \\
& \hat{P}_{28}=4 N^{9}+8 N^{8}+6 N^{7}+35 N^{6}+66 N^{5}-6 N^{4}-85 N^{3}-61 N^{2}-24 N-4 \text {. } \\
& \bar{A}_{t}^{Q g}=T_{F} C_{A}\left\{\frac{2}{3} \frac{N^{2}+3 N+4}{(N-1) N(N+1)^{2}(N+2)}\left(3 S_{3}+\zeta_{3}\right)\right. \\
& -\frac{2 N^{4}+5 N^{3}-3 N^{2}-20 N-16}{(N-1) N(N+1)^{3}(N+2)^{2}}\left(2 S_{2}+\zeta_{2}\right) \\
& \left.-\frac{\hat{P}_{29}}{(N-1) N(N+1)^{5}(N+2)^{4}}\right\} \text {, } \\
& \hat{P}_{29}=2 N^{8}+10 N^{7}+28 N^{6}+91 N^{5}+213 N^{4}+160 N^{3}-248 N^{2}-512 N-256 .
\end{aligned}
$$




$$
\begin{aligned}
A_{u}^{Q g}= & a_{s}^{2} S_{\varepsilon}^{2}\left\{\frac{8}{3 \varepsilon} T_{F}\left(1+\frac{\zeta_{2}}{8} \varepsilon^{2}\right) \sum_{i=1}^{3}\left(\frac{m_{i}^{2}}{\mu^{2}}\right)^{\varepsilon / 2}\right\}\left\{-8 T_{F}\left(\frac{m^{2}}{\mu^{2}}\right)^{\varepsilon / 2}\left(\frac{1}{\varepsilon} \frac{N^{2}+3 N+2}{N(N+1)(N+2)}\right.\right. \\
& \left.\left.-\frac{1}{(N+1)(N+2)}+\varepsilon\left(\frac{1}{2(N+1)(N+2)}+\frac{\zeta_{2}}{8 N}\right)\right)\right\} \\
A_{v}^{Q g}= & a_{s}^{2} S_{\varepsilon}^{2}\left\{\frac{8}{3 \varepsilon} T_{F}\left(1+\frac{\zeta_{2}}{8} \varepsilon^{2}\right) \sum_{i=1}^{3}\left(\frac{m_{i}^{2}}{\mu^{2}}\right)^{\varepsilon / 2}\right\} \\
& \times\left\{16 T_{F}\left(\frac{m^{2}}{\mu^{2}}\right)^{\varepsilon / 2}\left(\frac{1}{\varepsilon}-\frac{1}{2}+\varepsilon\left(\frac{1}{4}+\frac{\zeta_{2}}{8}\right)\right) \frac{1}{(N+1)(N+2)}\right\}
\end{aligned}
$$

Note that for diagram $u$ and $v$ the sum runs over all heavy quark flavors.

The diagrams contributing to the pure singlet contributions yield

$$
\begin{aligned}
\bar{A}_{a}^{Q q}= & T_{F} C_{F}\left\{-\frac{4}{3} \frac{(N+2)(N-1)}{N^{2}(N+1)^{2}}\left(3 S_{3}+\zeta_{3}\right)-2 \frac{5 N^{3}-5 N^{2}-16 N-4}{N^{3}(N+1)^{3}(N+2)}\left(2 S_{2}+\zeta_{2}\right)\right. \\
& \left.+\frac{2 \hat{P}_{30}}{N^{5}(N+1)^{5}(N+2)^{3}}\right\}, \\
\hat{P}_{30}= & 5 N^{9}+25 N^{8}-35 N^{7}-229 N^{6}-107 N^{5}+481 N^{4}+688 N^{3}+360 N^{2} \\
& +112 N+16 . \\
\bar{A}_{b}^{Q q}= & T_{F} C_{F}\left\{-\frac{32}{3} \frac{3 S_{3}+\zeta_{3}}{(N-1) N(N+1)(N+2)}\right. \\
& -32 \frac{2 N+3}{(N-1) N(N+1)^{2}(N+2)^{2}}\left(2 S_{2}+\zeta_{2}\right) \\
& \left.+32 \frac{(2 N+3)\left(N^{4}+6 N^{3}+5 N^{2}-12 N-16\right)}{N(N-1)(N+1)^{4}(N+2)^{4}}\right\} .
\end{aligned}
$$

The flavor non-singlet contributions are given by

$$
\begin{aligned}
\bar{A}_{a}^{q q, Q}= & T_{F} C_{F}\left\{-\frac{2}{9} \frac{(N+2)(N-1)}{N(N+1)} \zeta_{3}-\frac{2}{9} \frac{N^{4}+2 N^{3}-10 N^{2}-5 N+3}{N^{2}(N+1)^{2}} \zeta_{2}\right. \\
& \left.\quad-\frac{2}{81} \frac{\hat{P}_{31}}{N^{4}(N+1)^{4}}\right\}, \\
\hat{P}_{31}= & 49 N^{8}+196 N^{7}-83 N^{6}-533 N^{5}-374 N^{4}-59 N^{3}-33 N^{2}+9 N+27 . \\
\bar{A}_{b}^{q q, Q}= & T_{F} C_{F}\left\{\frac{4}{3} S_{4}+\frac{4}{3} S_{2} \zeta_{2}-\frac{8}{9} S_{1} \zeta_{3}-\frac{20}{9} S_{3}-\frac{20}{9} S_{1} \zeta_{2}+\frac{8}{9} \zeta_{3}+\frac{112}{27} S_{2}+\frac{8}{9} \zeta_{2}\right. \\
& \left.\quad-\frac{656}{81} S_{1}+\frac{392}{81}\right\}, \\
\bar{A}_{c}^{q q, Q}= & T_{F} C_{F}\left\{-\frac{\zeta_{2}}{2}-\frac{89}{72}\right\} .
\end{aligned}
$$




\section{Appendix B. Infinite sums}

In this appendix we list a series of infinite sums which were needed in the present analysis and are newly calculated. In addition we made use of the sums in [7]. $\sigma_{1}$ is a symbol for $\sum_{k=1}^{\infty}(1 / k)$ and the corresponding sums are divergent. The calculation was partly performed using integral representations, solving difference equations and using the summation package Sigma, see also [53].

\section{B.1. Weighted Beta functions}

$$
\begin{aligned}
& \sum_{i=1}^{\infty} \frac{B(N, i)}{(i+N+2)^{3}}=(-1)^{N}\left[\frac{4 S_{1,-2}(N+2)+2 S_{-3}(N+2)+2 \zeta_{2} S_{1}(N+2)+2 \zeta_{3}}{N(N+1)(N+2)}\right. \\
& \left.+\frac{-6 S_{-2}(N+2)-3 \zeta_{2}}{N(N+1)(N+2)}\right]+\frac{1}{N(N+1)(N+2)^{2}}, \\
& \sum_{i=1}^{\infty} \frac{B(N, i)}{(i+N+3)^{2}}=6(-1)^{N} \frac{2 S_{-2}(N+3)+\zeta_{2}}{N(N+1)(N+2)(N+3)} \\
& +\frac{N^{4}+5 N^{3}+13 N^{2}+18 N+13}{N(N+1)^{2}(N+2)^{2}(N+3)^{2}}, \\
& \sum_{i=1}^{\infty} \frac{B(N, i)}{(i+N+4)^{2}}=24(-1)^{N} \frac{2 S_{-2}(N+4)+\zeta_{2}}{N(N+1)(N+2)(N+3)(N+4)} \\
& +\frac{N^{6}+11 N^{5}+54 N^{4}+143 N^{3}+213 N^{2}+178 N+100}{N(N+1)^{2}(N+2)^{2}(N+3)^{2}(N+4)^{2}}, \\
& \sum_{i=1}^{\infty} \frac{B(N, i)}{(i+1)^{2}}=\frac{-1+2 N}{N}+(1-N) S_{1,2}(N)+\frac{-1+N+N^{2}}{N} S_{2}(N) \\
& +\left(\frac{1-N-N^{2}}{N}+(-1+N) S_{1}(N)\right) \zeta_{2}+(1-N) \zeta_{3}, \\
& \sum_{i=1}^{\infty} \frac{B(N, i)}{(i+4)}=-\frac{-144+300 N-415 N^{2}+241 N^{3}-63 N^{4}+6 N^{5}}{144 N^{2}} \\
& +\frac{(N-1)(N-2)(N-3)(N-4)}{24}\left(\zeta_{2}-S_{2}(N)\right) .
\end{aligned}
$$

B.2. Weighted Beta functions and harmonic sums

$$
\begin{aligned}
\sum_{i=1}^{\infty} \frac{B(N, i)}{i+1} S_{1}(i)= & (N-1) S_{3}(N-1)-(N-1) \zeta_{3}-S_{2}(N-1)+\zeta_{2}, \\
\sum_{i=1}^{\infty} \frac{B(N, i)}{i+2} S_{1}(i)= & \left(\zeta_{3}-S_{3}(N-1)\right) \frac{(N-1)(N-2)}{2} \\
& +\left(\zeta_{2}-S_{2}(N-1)\right) \frac{N^{2}-3 N+3}{2}+\frac{2-N}{2}
\end{aligned}
$$




$$
\begin{aligned}
\sum_{i=1}^{\infty} \frac{B(N, i)}{i+3} S_{1}(i)= & \left(-\zeta_{3}+S_{3}(N-1)\right) \frac{(N-1)(N-2)(N-3)}{6} \\
& -\left(\zeta_{2}-S_{2}(N-1)\right) \frac{3 N^{3}-18 N^{2}+33 N-22}{12} \\
& +\frac{(6 N-13)(N-3)}{24}, \\
\sum_{i=1}^{\infty} \frac{B(N, i)}{i+4} S_{1}(i)= & -\frac{-864+2040 N^{2}-3746 N^{3}+2453 N^{4}-675 N^{5}+66 N^{6}}{864 N^{3}} \\
& +\frac{300-550 N+385 N^{2}-110 N^{3}+11 N^{4}}{144}\left(\zeta_{2}-S_{2}(N)\right) \\
& +\frac{(N-1)(N-2)(N-3)(N-4)}{24}\left(\zeta_{3}-S_{3}(N)\right),
\end{aligned}
$$

$\sum_{i=1}^{\infty} \frac{B(N, i)}{i+N+3} S_{1}(i)$

$$
=\frac{\zeta_{2}-S_{2}(N+2)}{N+3}+\frac{3 N^{6}+15 N^{5}+36 N^{4}+51 N^{3}+52 N^{2}+36 N+8}{N^{3}(N+1)^{3}(N+2)^{3}},
$$

$$
\sum_{i=1}^{\infty} \frac{B(N, i)}{i+N+4} S_{1}(i)
$$$$
=\frac{\zeta_{2}-S_{2}(N+3)}{N+4}+2 \frac{2 N^{9}+24 N^{8}+129 N^{7}+408 N^{6}+854 N^{5}+1270 N^{4}}{N^{3}(N+1)^{3}(N+2)^{3}(N+3)^{3}}
$$

$$
\begin{array}{r}
+2 \frac{1405 N^{3}+1158 N^{2}+594 N+108}{N^{3}(N+1)^{3}(N+2)^{3}(N+3)^{3}}, \\
\sum_{i=1}^{\infty} \frac{B(N, i)}{(i+N+1)^{2}} S_{1}(i)
\end{array}
$$

$$
=(-1)^{N} \frac{2 S_{1,-2}(N)+S_{-3}(N)+\zeta_{2} S_{1}(N)+\zeta_{3}}{N(N+1)}+\frac{\zeta_{2}-S_{2}(N)}{(N+1)^{2}},
$$

$$
\begin{aligned}
\sum_{i=1}^{\infty} & \frac{B(N, i)}{i+1} S_{1}(i+N) \\
= & (N-1)\left(2 S_{3}(N)+S_{1}(N) S_{2}(N)-\zeta_{2} S_{1}(N)-2 \zeta_{3}\right)-\frac{N-1}{N}\left(S_{2}(N)-\zeta_{2}\right) \\
& \quad+\frac{1-N+N^{2}}{N^{2}} S_{1}(N)+\frac{1}{N^{3}},
\end{aligned}
$$$$
\sum_{i=1}^{\infty} \frac{B(N, i)}{i+2} S_{1}(i+N)
$$$$
=\frac{(N-1)(N-2)}{2}\left(-2 S_{3}(N)-S_{1}(N) S_{2}(N)+\zeta_{2} S_{1}(N)+2 \zeta_{3}\right)
$$ 


$$
\begin{aligned}
& +\frac{(N-2)(2 N-1)}{2 N}\left(S_{2}(N)-\zeta_{2}\right)-\frac{-4+6 N-7 N^{2}+2 N^{3}}{4 N^{2}} S_{1}(N) \\
& +\frac{2-2 N^{2}+N^{3}}{2 N^{3}} \\
& \sum_{i=1}^{\infty} \frac{B(N, i)}{i+3} S_{1}(i+N) \\
& =\frac{(N-1)(N-2)(N-3)}{6}\left(2 S_{3}(N)+S_{1}(N) S_{2}(N)-\zeta_{2} S_{1}(N)-2 \zeta_{3}\right) \\
& -\frac{(N-3)\left(3 N^{2}-6 N+2\right)}{6 N}\left(S_{2}(N)-\zeta_{2}\right)-\frac{-12+21 N^{2}-19 N^{3}+4 N^{4}}{12 N^{3}} \\
& +\frac{36-66 N+85 N^{2}-39 N^{3}+6 N^{4}}{36 N^{2}} S_{1}(N), \\
& \sum_{i=1}^{\infty} \frac{B(N, i)}{i+4} S_{1}(i+N) \\
& =\frac{144-340 N^{2}+397 N^{3}-150 N^{4}+18 N^{5}}{144 N^{3}} \\
& -\frac{-144+300 N-415 N^{2}+241 N^{3}-63 N^{4}+6 N^{5}}{144 N^{2}} S_{1}(N) \\
& +\left\{-\frac{(N-4)(2 N-3)\left(1-3 N+N^{2}\right)}{12 N}\right. \\
& \left.+\frac{(N-1)(N-2)(N-3)(N-4)}{24} S_{1}(N)\right\}\left(\zeta_{2}-S_{2}(N)\right) \\
& +\frac{(N-1)(N-2)(N-3)(N-4)}{12}\left(\zeta_{3}-S_{3}(N)\right), \\
& \sum_{i=1}^{\infty} \frac{B(N, i)}{(i+N+1)^{2}} S_{1}(i+N) \\
& =\frac{(-1)^{N+1}}{N(N+1)}\left(4 S_{1,-2}(N+1)-2 S_{-3}(N+1)\right. \\
& \left.-2 S_{1}(N+1) S_{-2}(N+1)+\zeta_{2} S_{1}(N+1)-\zeta_{3}-2 S_{-2}(N+1)-\zeta_{2}\right) \\
& +\frac{S_{1}(N+1)}{N(N+1)^{2}}+\frac{1}{N(N+1)^{3}} \text {, } \\
& \sum_{i=1}^{\infty} \frac{B(N, i)}{(i+N+2)^{2}} S_{1}(i+N) \\
& =\frac{(-1)^{N+1}}{N(N+1)(N+2)}\left(8 S_{1,-2}(N+2)-4 S_{-3}(N+2)-4 S_{1}(N+2) S_{-2}(N+2)\right. \\
& \left.+2 \zeta_{2} S_{1}(N+2)-2 \zeta_{3}-10 S_{-2}(N+2)-5 \zeta_{2}\right) \\
& +\frac{1+N+N^{2}}{N(N+1)^{2}(N+2)^{2}} S_{1}(N+2)-\frac{1+7 N+6 N^{2}+N^{3}}{N(N+1)^{3}(N+2)^{3}},
\end{aligned}
$$




$$
\begin{aligned}
& \sum_{i=1}^{\infty} \frac{B(N, i)}{(i+N+3)} S_{1}(i+N) \\
& =\frac{16 N^{3}+12+40 N+30 N^{2}+6 N^{4}+N^{5}}{N^{3}(N+1)^{2}(N+2)^{2}(N+3)} \\
& +\frac{85 N^{2}+36+66 N+69 N^{3}+34 N^{4}+9 N^{5}+N^{6}}{N^{2}(N+1)^{2}(N+2)^{2}(N+3)^{2}} S_{1}(N) \\
& -6(-1)^{N}\left(\frac{\zeta(2)+2 S_{-2}(N)}{N(N+1)(N+2)(N+3)}\right) \\
& \sum_{i=1}^{\infty} \frac{B(N, i)}{(i+N+4)} S_{1}(i+N) \\
& =\frac{144+564 N+564 N^{2}+361 N^{3}+180 N^{4}+62 N^{5}+12 N^{6}+N^{7}}{N^{3}(N+1)^{2}(N+2)^{2}(N+3)^{2}(N+4)} \\
& +\frac{424 N^{5}+110 N^{6}+16 N^{7}+N^{8}}{N^{2}(N+1)^{2}(N+2)^{2}(N+3)^{2}(N+4)^{2}} S_{1}(N) \\
& +\frac{576+1200 N+1660 N^{2}+1576 N^{3}+1013 N^{4}}{N^{2}(N+1)^{2}(N+2)^{2}(N+3)^{2}(N+4)^{2}} S_{1}(N) \\
& -24(-1)^{N}\left(\frac{\zeta(2)+2 S_{-2}(N)}{N(N+1)(N+2)(N+3)(N+4)}\right) \text {, } \\
& \sum_{i=1}^{\infty} B(N, i) S_{1}(i+N)^{2} \\
& =\frac{-2+5 N-2 N^{2}+N^{3}}{(N-1)^{3} N^{3}}+2 \frac{\left(1-N+N^{2}\right) S_{1}(N)}{(N-1)^{2} N^{2}}+\frac{S_{1}(N)^{2}}{N-1} \\
& +2 \frac{(-1)^{N} S_{-2}(N)}{(N-1) N}+\frac{(-1)^{N} \zeta_{2}}{(N-1) N}, \\
& \sum_{i=1}^{\infty} \frac{B(N, i)}{i} S_{1}(i+N)^{2} \\
& =\frac{2}{N^{4}}+2 \frac{S_{1}(N)}{N^{3}}+\frac{S_{1}^{2}(N)}{N^{2}}-S_{1}^{2}(N) S_{2}(N)-4 S_{1}(N) S_{3}(N)-3 S_{4}(N) \\
& -2 \frac{(-1)^{N} S_{-2}(N)}{N^{2}}+2 S_{-2}^{2}(N)-\frac{(-1)^{N} \zeta_{2}}{N^{2}}+S_{1}^{2}(N) \zeta_{2}+2 S_{-2}(N) \zeta_{2} \\
& +\frac{17}{10} \zeta_{2}^{2}+4 S_{1}(N) \zeta_{3} \\
& \sum_{i=1}^{\infty} \frac{B(N, i)}{i+N} S_{1}(i+N)^{2} \\
& =\frac{(-1)^{N+1}}{N^{2}}\left(\zeta_{2}+2 S_{-2}(N)\right)+\frac{S_{1}(N)^{2}}{N^{2}}+2 \frac{S_{1}(N)}{N^{3}}+\frac{2}{N^{4}},
\end{aligned}
$$




$$
\begin{aligned}
\sum_{i=1}^{\infty} & \frac{B(N, i)}{i+N+1} S_{1}(i+N)^{2} \\
= & \frac{(-1)^{N}}{N(N+1)}\left(-6 S_{-2,1}(N-1)+2 S_{-2}(N-1) S_{1}(N-1)+S_{-3}(N-1)\right. \\
& \left.+\zeta_{2} S_{1}(N-1)-3 \zeta_{3}-2 S_{-2}(N-1)-\zeta_{2}\right)+\frac{1+N+N^{2}}{N^{2}(N+1)^{2}} S_{1}(N-1)^{2} \\
& +2 \frac{1+2 N}{N^{2}(N+1)^{2}} S_{1}(N-1)+\frac{2+3 N}{N^{3}(N+1)^{2}}, \\
\sum_{i=1}^{\infty} \frac{B(N, i)}{i}+N+2 S_{1}(i+N)^{2} & \frac{(-1)^{N}}{N(N+1)(N+2)}\left(-12 S_{-2,1}(N-1)+4 S_{-2}(N-1) S_{1}(N-1)\right. \\
& \left.+2 S_{-3}(N-1)+2 \zeta_{2} S_{1}(N-1)-6 \zeta_{3}+2(N-1) S_{-2}(N-1)+(N-5) \zeta_{2}\right) \\
& +\frac{4+6 N+7 N^{2}+4 N^{3}+N^{4}}{N^{2}(N+1)^{2}(N+2)^{2}} S_{1}(N-1)^{2}+4 \frac{3+3 N+N^{2}}{N^{2}(N+1)(N+2)^{2}} S_{1}(N-1) \\
& +\frac{4+11 N+5 N^{2}}{N^{3}(N+1)(N+2)^{2}}, \\
\sum_{i=1}^{\infty} \frac{B(N, i)}{i+N} S_{1}(i)^{2}=\frac{S_{1,2}(N-1)-2 S_{3}(N-1)-\zeta_{2} S_{1}(N-1)+3 \zeta_{3}}{i+N+1} & \frac{B(N, i)}{S_{1,2}(N)-2 S_{3}(N)-\zeta_{2} S_{1}(N)+3 \zeta_{3}}-\frac{S_{2}(N)-\zeta_{2}}{N^{2}}+\frac{2}{N^{4}},
\end{aligned}
$$$$
\sum_{i=1}^{\infty} \frac{B(N, i)}{i+N+2} S_{1}(i)^{2}
$$$$
=\frac{S_{1,2}(N+1)-2 S_{3}(N+1)-\zeta_{2} S_{1}(N+1)+3 \zeta_{3}}{N+2}-\frac{2 N^{2}+N+1}{N^{2}(N+1)^{2}}\left(S_{2}(N+1)-\zeta_{2}\right)
$$$$
+\frac{5 N^{4}+8 N^{3}+13 N^{2}+8 N+2}{N^{4}(N+1)^{4}},
$$

$$
\begin{aligned}
\sum_{i=1}^{\infty} & \frac{B(N, i)}{i+N+2} S_{1}(i) S_{1}(N+i) \\
= & \frac{(-1)^{N}}{N(N+1)(N+2)}\left(4 S_{-2,1}(N)-6 S_{-3}(N)-4 S_{-2}(N) S_{1}(N)-2 \zeta_{2} S_{1}(N)-2 \zeta_{3}\right. \\
& \left.-2 \frac{\zeta_{2}}{(N+1)}-4 \frac{S_{-2}(N)}{(N+1)}\right)-2 \frac{S_{3}(N)}{N+2}-\frac{S_{1}(N) S_{2}(N)}{N+2}+\frac{\zeta_{2} S_{1}(N)}{N+2}+\frac{2 \zeta_{3}}{N+2} \\
& +\frac{2+7 N+7 N^{2}+5 N^{3}+N^{4}}{N^{3}(N+1)^{3}(N+2)} S_{1}(N)+2 \frac{2+7 N+9 N^{2}+4 N^{3}+N^{4}}{N^{4}(N+1)^{3}(N+2)},
\end{aligned}
$$




$$
\begin{aligned}
& \sum_{i=1}^{\infty} B(N, i) S_{2}(N+i)=\frac{1}{(N-1) N^{2}}+\frac{S_{2}(N)}{N-1}-2 \frac{(-1)^{N} S_{-2}(N)}{(N-1) N}-\frac{(-1)^{N} \zeta_{2}}{(N-1) N} \\
& \sum_{i=1}^{\infty} \frac{B(N, i)}{i} S_{2}(N+i) \\
& =-3 S_{4}(N)-2 S_{-2}(N)^{2}-S_{2}(N)^{2}-2 S_{-2}(N) \zeta_{2}+S_{2}(N) \zeta_{2}+\frac{7}{10} \zeta_{2}^{2} \\
& \quad+(-1)^{N} \frac{\zeta(2)+2 S_{-2}(N)}{N^{2}}+\frac{S_{2}(N)}{N^{2}} \\
& \sum_{i=1}^{\infty} \frac{B(N, i)}{N+i} S_{2}(N+i)=(-1)^{N} \frac{\zeta(2)+2 S_{-2}(N)}{N^{2}}+\frac{S_{2}(N)}{N^{2}} \\
& \sum_{i=1}^{\infty} \frac{B(N, i)}{N+i+1} S_{2}(N+i) \\
& =\frac{1+N+N^{2}}{N^{2}(N+1)^{2}} S_{2}(N)+(-1)^{N}\left[-\frac{2 S_{1,-2}(N)}{N(N+1)}+\frac{2 S_{-2}(N)}{N^{2}}\right. \\
& \left.\quad-\frac{S_{-3}(N)}{N(N+1)}+\frac{\zeta_{2}}{N^{2}}-\frac{S_{1}(N) \zeta_{2}}{N(N+1)}-\frac{\zeta_{3}}{N(N+1)}\right]
\end{aligned}
$$$$
\sum_{i=1}^{\infty} \frac{B(N, i)}{N+i+2} S_{2}(N+i)
$$$$
=\frac{N^{4}+4 N^{3}+7 N^{2}+6 N+4}{N^{2}(N+1)^{2}(N+2)^{2}} S_{2}(N)+\frac{2}{N(N+1)^{2}(N+2)}
$$$$
+(-1)^{N}\left[-\frac{4 S_{1,-2}(N)}{N(N+1)(N+2)}-\frac{2(N-2) S_{-2}(N)}{N^{2}(N+2)}-\frac{2 S_{-3}(N)}{N(N+1)(N+2)}\right.
$$$$
\left.+\zeta_{2}\left(-\frac{2 S_{1}(N)}{N(N+1)(N+2)}-\frac{N-2}{N^{2}(N+2)}\right)-\frac{2 \zeta_{3}}{N(N+1)(N+2)}\right] \text {, }
$$

$\sum_{i=1}^{\infty} \frac{B(N, i)}{N+i+2} S_{2}(i)$

$$
\begin{aligned}
= & \frac{S_{1,2}(N)}{N+2}-\frac{S_{1}(N) \zeta_{2}}{N+2}+\frac{\zeta_{3}}{N+2}-\frac{\left(2+3 N+4 N^{2}+N^{3}\right) S_{2}(N)}{N^{2}(N+1)^{2}(N+2)} \\
& +\frac{\left(2+3 N+4 N^{2}+N^{3}\right) \zeta_{2}}{N^{2}(N+1)^{2}(N+2)}+\frac{2}{N(N+1)^{4}(N+2)},
\end{aligned}
$$

$$
\begin{aligned}
& \sum_{i=1}^{\infty} \frac{B(N, i)}{(N+i+2)^{2}} S_{1}(i) \\
& \quad=\frac{-8+N+3 N^{2}}{N(N+1)^{4}(N+2)^{2}}-\frac{S_{2}(N)}{(N+2)^{2}}+\frac{\zeta_{2}}{(N+2)^{2}}
\end{aligned}
$$




$$
\begin{aligned}
& +\left(\frac{2 \zeta_{2}}{N(1+N)^{2}(N+2)}+\frac{2 S_{1}(N) \zeta_{2}}{N(N+1)(N+2)}+\frac{2 \zeta_{3}}{N(N+1)(N+2)}\right. \\
& \left.+\frac{4 S_{-2}(N)}{N(N+1)^{2}(N+2)}+\frac{2 S_{-3}(N)}{N(N+1)(N+2)}+\frac{4 S_{1,-2}(N)}{N(N+1)(N+2)}\right)(-1)^{N} .
\end{aligned}
$$

\section{B.3. Weighted harmonic sums}

$$
\begin{aligned}
\sum_{i=1}^{\infty} \frac{S_{1}(i) S_{1}(i+N)}{i+N}= & \frac{\sigma_{1}^{3}-\zeta_{3}-S_{1}^{3}(N)-2 S_{3}(N)}{3}+\frac{S_{1}^{2}(N)+S_{2}(N)}{N} \\
& -S_{1}(N) S_{2}(N) \\
\sum_{i=1}^{\infty} \frac{S_{1}(i) S_{1}(i+N)}{i+N+1}= & \frac{\sigma_{1}^{3}-4 \zeta_{3}}{3}+\frac{S_{3}(N)-S_{1}^{3}(N)}{3}-S_{1}(N) \zeta_{2} \\
\sum_{i=1}^{\infty} \frac{S_{1}(i) S_{1}(i+N)}{i+N+2}= & \frac{\sigma_{1}^{3}-4 \zeta_{3}}{3}+\frac{S_{3}(N)-S_{1}^{3}(N)}{3}-\frac{S_{1}^{2}(N)}{N+1}-S_{1}(N) \zeta_{2}-\frac{\zeta_{2}}{N+1} \\
& -\frac{(N+2)}{(N+1)^{2}} S_{1}(N)-\frac{1}{(N+1)^{2}}, \\
\sum_{i=1}^{\infty} \frac{S_{1}(i) S_{1}(i+N)}{i+3}= & \frac{\sigma_{1}^{3}-\zeta_{3}}{3}+S_{1,1,1}(N)-\frac{3 N^{2}-6 N+2}{N(N-1)(N-2)} S_{1,1}(N) \\
& +\frac{13 N-19}{4(N-1)(N-2)}-\frac{(N+1)(7 N-6)}{4 N(N-1)} S_{1}(N) \\
\sum_{i=1}^{\infty} \frac{S_{1}(i) S_{1}(i+N)}{i+4}= & \frac{1}{3} \sigma_{1}^{3}-\frac{(N+1)\left(132-232 N+85 N^{2}\right)}{36(N-2)(N-1) N} S_{1}(N)-\frac{1}{3} \zeta_{3}+S_{1,1,1}(N) \\
& +\frac{(-3+2 N)\left(1-3 N+N^{2}\right)}{(N-3)(N-2)(N-1) N} S_{1,1}(N) \\
& +\frac{809-909 N+232 N^{2}}{36(N-3)(N-2)(N-1)},
\end{aligned}
$$

$\sum_{i=1}^{\infty} \frac{S_{1}(i+N) S_{1}^{2}(i)}{i}=\frac{\sigma_{1}^{4}}{4}+\frac{43}{20} \zeta_{2}^{2}+3 S_{1}(N) \zeta_{3}+\frac{S_{1}^{2}(N)-S_{2}(N)}{2} \zeta_{2}-S_{1}(N) S_{2,1}(N)$

$$
+\frac{S_{1}^{2}(N) S_{2}(N)}{2}+\frac{2}{3} S_{1}(N) S_{3}(N)-\frac{S_{2}^{2}(N)}{4}+\frac{S_{1}^{4}(N)}{12},
$$

$$
\begin{aligned}
\sum_{i=1}^{\infty} \frac{S_{1}^{2}(i+N) S_{1}(i)}{i}= & \frac{\sigma_{1}^{4}}{4}+\frac{43}{20} \zeta_{2}^{2}+5 S_{1}(N) \zeta_{3}+\frac{3 S_{1}^{2}(N)-S_{2}(N)}{2} \zeta_{2}-2 S_{1}(N) S_{2,1}(N) \\
& +S_{1}^{2}(N) S_{2}(N)+S_{1}(N) S_{3}(N)-\frac{S_{2}^{2}(N)}{4}+\frac{S_{1}^{4}(N)}{4} \\
\sum_{i=1}^{\infty} \frac{S_{1}(i) S_{2}(i+N)}{i}= & \frac{\sigma_{1}^{2}}{2} \zeta_{2}-\frac{1}{5} \zeta_{2}^{2}-S_{1}(N) \zeta_{3}-\frac{S_{1}^{2}(N)-3 S_{2}(N)}{2} \zeta_{2}-2 S_{3,1}(N)
\end{aligned}
$$$$
+\frac{1}{2} S_{4}(N)+\frac{S_{1}^{2}(N) S_{2}(N)}{2}+S_{1}(N) S_{3}(N),
$$ 


$$
\begin{aligned}
\sum_{i=1}^{\infty} \frac{S_{1}(i+N) S_{2}(i)}{i}= & \frac{\sigma_{1}^{2}}{2} \zeta_{2}-\frac{\zeta_{2}^{2}}{5}+S_{1}(N) \zeta_{3}+\frac{S_{1}^{2}(N)-S_{2}(N)}{2} \zeta_{2}+2 S_{1,1,2}(N) \\
& -2 S_{1,3}(N)+S_{1}(N) S_{2,1}(N)-S_{1}^{2}(N) S_{2}(N) \\
& +\frac{S_{4}(N)-S_{2}^{2}(N)}{2},
\end{aligned}
$$

$$
\begin{aligned}
\sum_{i=1}^{\infty} & \frac{S_{1,1}(i+N) S_{1}(i)}{i+N} \\
= & \frac{\sigma_{1}^{4}}{8}+\frac{\sigma_{1}^{2}}{4} \zeta_{2}-\frac{9}{40} \zeta_{2}^{2}-3 \frac{S_{4}(N)}{4}+\frac{S_{1}^{3}(N)}{2 N}-\frac{S_{1}^{4}(N)}{8}-3 \frac{S_{1}^{2}(N) S_{2}(N)}{4} \\
& -3 \frac{S_{2}^{2}(N)}{8}+S_{1}(N)\left(3 \frac{S_{2}(N)}{2 N}-S_{3}(N)\right)+\frac{S_{3}(N)}{N}
\end{aligned}
$$

$$
\begin{aligned}
\sum_{i=1}^{\infty} \frac{S_{1,1}(i+N) S_{1}(i)}{i}= & \frac{\sigma_{1}^{4}}{8}+\frac{\sigma_{1}^{2} \zeta_{2}}{4}+\frac{39}{40} \zeta_{2}^{2}+2 S_{1}(N) \zeta_{3}+\frac{S_{1}^{2}(N)+S_{2}(N)}{2} \zeta_{2} \\
& -S_{3,1}(N)+3 \frac{S_{1}^{2}(N) S_{2}(N)}{4}+\frac{S_{1}^{4}(N)}{8}-\frac{S_{2}^{2}(N)}{8} \\
& +S_{1}(N)\left(-S_{2,1}(N)+S_{3}(N)\right)+\frac{S_{4}(N)}{4}
\end{aligned}
$$

$$
\begin{aligned}
\sum_{i=1}^{\infty} \frac{S_{1}(i) S_{2}(i+N)}{i+N}= & \frac{\sigma_{1}^{2}}{2} \zeta_{2}-\frac{9}{10} \zeta_{2}^{2}+\left(-\frac{S_{1}(N)}{N}+\frac{S_{1}^{2}(N)}{2}+\frac{S_{2}(N)}{2}\right) \zeta_{2}-S_{1}^{2}(N) S_{2}(N) \\
& -\frac{S_{2}^{2}(N)}{2}-\frac{S_{2,1}(N)}{N}+S_{1}(N)\left(2 \frac{S_{2}(N)}{N}+S_{2,1}(N)-2 S_{3}(N)\right) \\
& +2 \frac{S_{3}(N)}{N}+S_{3,1}(N)-\frac{3 S_{4}(N)}{2}
\end{aligned}
$$

$$
\begin{aligned}
& \sum_{i=1}^{\infty} \frac{S_{2}(i) S_{1}(i+N)}{i+N} \\
& \quad=\frac{\sigma_{1}^{2}}{2} \zeta_{2}-\frac{7}{10} \zeta_{2}^{2}+\left(\frac{2}{N}-2 S_{1}(N)\right) \zeta_{3}+\left(\frac{S_{1}(N)}{N}-\frac{S_{1}^{2}(N)}{2}-\frac{S_{2}(N)}{2}\right) \zeta_{2}
\end{aligned}
$$$$
-\frac{S_{1}^{2}(N)}{N^{2}}-\frac{S_{2}(N)}{N^{2}}+\frac{S_{2}^{2}(N)}{2}-\frac{S_{2,1}(N)}{N}+S_{1}(N) S_{2,1}(N)
$$$$
+S_{3,1}(N)+\frac{S_{4}(N)}{2},
$$

$$
\begin{aligned}
\sum_{i=1}^{\infty} & \frac{S_{1}(i+N) S_{1}(i)}{(i+N)^{2}} \\
= & \frac{6}{5} \zeta_{2}^{2}+\left(-\frac{2}{N}+2 S_{1}(N)\right) \zeta_{3}+\frac{S_{1}^{2}(N)}{N^{2}}+\frac{S_{2}(N)}{N^{2}}-\frac{S_{2}^{2}(N)}{2}+\frac{S_{2,1}(N)}{N} \\
& -S_{1}(N) S_{2,1}(N)-S_{3,1}(N)-\frac{S_{4}(N)}{2}
\end{aligned}
$$




$$
\begin{aligned}
\sum_{i=1}^{\infty} & \frac{S_{1}(i+N) S_{1}^{2}(i)}{i+N} \\
= & \frac{\sigma_{1}^{4}}{4}-\frac{3 \zeta_{2}^{2}}{4}+\left(\frac{2}{N}-2 S_{1}(N)\right) \zeta_{3}+\left(\frac{S_{1}(N)}{N}-\frac{S_{1}^{2}(N)}{2}-\frac{S_{2}(N)}{2}\right) \zeta_{2}+\frac{S_{1}^{3}(N)}{N} \\
& \quad-\frac{S_{1}^{4}(N)}{4}+S_{1}^{2}(N)\left(-\frac{1}{N^{2}}-\frac{3 S_{2}(N)}{2}\right)-\frac{S_{2}(N)}{N^{2}}-\frac{S_{2}^{2}(N)}{4}-\frac{S_{2,1}(N)}{N} \\
& +S_{1}(N)\left(3 \frac{S_{2}(N)}{N}+S_{2,1}(N)-2 S_{3}(N)\right)+2 \frac{S_{3}(N)}{N}+S_{3,1}(N)-S_{4}(N) .
\end{aligned}
$$

\section{B.4. Harmonic sums}

$$
\begin{aligned}
& \sum_{i=1}^{\infty} S_{3}(i+N)-S_{3}(i)=S_{2}(N)-(N+1) S_{3}(N)+N \zeta_{3}, \\
& \sum_{i=1}^{\infty}\left(S_{3}(i+N)-S_{3}(i)\right) i \\
& =\frac{S_{1}(N)-S_{2}(N)+N(N+1) S_{3}(N)-N(N+1) \zeta_{3}}{2}-N S_{2}(N)+N \zeta_{2}, \\
& \sum_{i=1}^{\infty}\left(S_{1}(i+N)-S_{1}(i)\right)^{3} \\
& =-\frac{3}{2} S_{1}^{2}(N)-S_{1}^{3}(N)-\frac{1}{2} S_{2}(N)+3 N S_{2,1}(N)-N S_{3}(N)+N \zeta_{3}, \\
& \sum_{i=1}^{\infty}\left(S_{1}(i+N)-S_{1}(i)\right)^{3} i \\
& =\frac{N}{2}\left(-(N+1) \zeta_{3}-3(N+1) S_{2,1}(N)+(N+1) S_{3}(N)+(3 N-1) \zeta_{2}\right) \\
& +\frac{1+2 N+6 N^{2}}{4} S_{2}(N)+\frac{3}{4} S_{1}^{2}(N)+\frac{2-6 N}{4} S_{1}(N), \\
& \sum_{i=1}^{\infty}\left(S_{1}(i+N)-S_{1}(i)\right) S_{2}(i) \\
& =-\frac{1}{2} S_{1}^{2}(N)-\frac{1}{2} S_{2}(N)+N S_{2,1}(N)+N \zeta_{2}-N S_{1}(N) \zeta_{2}+N \sigma_{1} \zeta_{2}-2 N \zeta_{3}, \\
& \sum_{i=1}^{\infty}\left(S_{1}(i+N)-S_{1}(i)\right) S_{2}(i+N) \\
& =-\left(1+S_{1}(N)\right) S_{2}(N)+N S_{3}(N)-N S_{1}(N) \zeta_{2}+N\left(\zeta_{2}+\sigma_{1} \zeta_{2}-\zeta_{3}\right) .
\end{aligned}
$$

\section{B.5. Miscellaneous sums}

$$
\sum_{k=0}^{l-1} \frac{B(k+2, \varepsilon / 2)}{k+1}=\frac{4}{\varepsilon^{2}}-\frac{2}{\varepsilon} B\left(1+l, \frac{\varepsilon}{2}\right),
$$




$$
\sum_{l=0}^{N-1}\left(\begin{array}{c}
N-1 \\
l
\end{array}\right)(-1)^{l} B\left(l+1-\frac{\varepsilon}{2}, 2-\frac{\varepsilon}{2}\right)=B\left(1-\frac{\varepsilon}{2}, N+1-\frac{\varepsilon}{2}\right) .
$$

\section{B.6. Double and other sums}

$$
\begin{aligned}
& \sum_{i, j=1}^{\infty} \frac{S_{1}(i) S_{1}(i+j+N)}{i(i+j)(j+N)} \\
& =6 \frac{S_{1}(N)}{N} \zeta_{3}+\zeta_{2}\left(2 \frac{S_{1}^{2}(N)}{N}+\frac{S_{2}(N)}{N}\right)+\frac{S_{1}^{4}(N)}{6 N}+\frac{S_{1}^{2}(N) S_{2}(N)}{N}-\frac{S_{2}^{2}(N)}{N} \\
& +4 \frac{S_{2,1,1}(N)}{N}+S_{1}(N)\left(-3 \frac{S_{2,1}(N)}{N}+4 \frac{S_{3}(N)}{3 N}\right)-2 \frac{S_{3,1}(N)}{N}-\frac{S_{4}(N)}{2 N}, \\
& \sum_{k=1}^{\infty} \frac{B(k+\varepsilon / 2, N)}{N+k} \\
& =\frac{1}{N^{2}}+\frac{\varepsilon}{2}\left\{(-1)^{N} \frac{2 S_{-2}(N)+\zeta_{2}}{N}-\frac{S_{1}(N)}{N^{2}}\right\} \\
& +\varepsilon^{2}\left\{-(-1)^{N} \frac{S_{-2,1}(N)}{N}+(-1)^{N} \frac{2 S_{-3}(N)-\zeta_{3}}{4 N}\right. \\
& \left.+(-1)^{N} \frac{2 S_{-2}(N)+\zeta_{2}}{4 N} S_{1}(N)+\frac{S_{1}^{2}(N)+S_{2}(N)}{8 N^{2}}\right\} \\
& +\frac{\varepsilon^{3}}{48 N}\left\{\frac{12}{5}(-1)^{N} \zeta_{2}^{2}-6(-1)^{N} S_{1}(N) \zeta_{3}+\zeta_{2}\left(3(-1)^{N} S_{1}^{2}(N)+3(-1)^{N} S_{2}(N)\right)\right. \\
& +48(-1)^{N} S_{-2,1,1}(N)-24(-1)^{N} S_{-3,1}(N)-24(-1)^{N} S_{-2,2}(N) \\
& +12(-1)^{N} S_{-4}(N)+6(-1)^{N} S_{1}^{2}(N) S_{-2}(N)+6(-1)^{N} S_{2}(N) S_{-2}(N)-2 \frac{S_{3}(N)}{N} \\
& \left.-\frac{S_{1}^{3}(N)}{N}+S_{1}(N)\left(-3 \frac{S_{2}(N)}{N}-24(-1)^{N} S_{-2,1}(N)+12(-1)^{N} S_{-3}(N)\right)\right\} \\
& +O\left(\varepsilon^{4}\right) \\
& \sum_{k=1}^{\infty} \frac{B(N+1, k+\varepsilon / 2)}{N+k} \\
& =(-1)^{N} \sum_{j=1}^{N}(-1)^{j}\left(\sum_{k=1}^{\infty} \frac{B(j, k+\varepsilon / 2)}{j+k}+\frac{B(j, 1+\varepsilon / 2)}{j}\right) \\
& +(-1)^{N} \sum_{k=1}^{\infty} \frac{B(1, k+\varepsilon / 2)}{k}, \\
& \sum_{k=1}^{\infty} \frac{B(k+\varepsilon / 2, N+1)}{N+k} \\
& =(-1)^{N}\left[2 S_{-2}(N)+\zeta_{2}\right]+\frac{\varepsilon}{2}(-1)^{N}\left[-\zeta_{3}+\zeta_{2} S_{1}(N)\right.
\end{aligned}
$$




$$
\begin{aligned}
& \left.+2 S_{1,-2}(N)-2 S_{-2,1}(N)\right]+\frac{\varepsilon^{2}}{4}(-1)^{N}\left[\frac{2}{5} \zeta_{2}^{2}-\zeta_{3} S_{1}(N)+\zeta_{2} S_{1,1}(N)\right. \\
& \left.+2\left\{S_{1,1,-2}(N)+S_{-2,1,1}(N)-S_{1,-2,1}(N)\right\}\right] \\
& +\varepsilon^{3}(-1)^{N}\left[-\frac{\zeta_{5}}{8}+\frac{S_{1}(N)}{20} \zeta_{2}^{2}-\frac{S_{1,1}(N)}{8} \zeta_{3}+\frac{S_{1,1,1}(N)}{8} \zeta_{2}\right. \\
& \left.+\frac{S_{1,-2,1,1}(N)+S_{1,1,1,-2}(N)-S_{-2,1,1,1}(N)-S_{1,1,-2,1}(N)}{4}\right]+O\left(\varepsilon^{4}\right) .
\end{aligned}
$$

\section{B.7. Expansion of harmonic sums for small argument}

One may expand nested harmonic sums into Taylor series w.r.t. the outer argument, using the corresponding differentiation rules [56]. In the present calculation we made use of the following relations.

$$
\begin{aligned}
& S_{1}(\varepsilon)=\zeta_{2} \varepsilon-\zeta_{3} \varepsilon^{2}+\frac{2}{5} \zeta_{2}^{2} \varepsilon^{3}-\zeta_{5} \varepsilon^{4}+O\left(\varepsilon^{5}\right) \\
& S_{2}(\varepsilon)=2 \zeta_{3} \varepsilon-\frac{6}{5} \zeta_{2}^{2} \varepsilon^{2}+4 \zeta_{5} \varepsilon^{3}-\frac{8}{7} \zeta_{2}^{3} \varepsilon^{4}+O\left(\varepsilon^{5}\right) \\
& S_{3}(\varepsilon)=\frac{6}{5} \zeta_{2}^{2} \varepsilon-6 \zeta_{5} \varepsilon^{2}+\frac{16}{7} \zeta_{2}^{3} \varepsilon^{3}-15 \zeta_{7} \varepsilon^{4}+O\left(\varepsilon^{5}\right), \\
& S_{4}(\varepsilon)=4 \zeta_{5} \varepsilon-\frac{16}{7} \zeta_{2}^{3} \varepsilon^{2}+20 \zeta_{7} \varepsilon^{3}-\frac{24}{5} \zeta_{2}^{4} \varepsilon^{4}+O\left(\varepsilon^{5}\right), \\
& S_{2,1}(\varepsilon)=\frac{7}{10} \zeta_{2}^{2} \varepsilon+\left(2 \zeta_{3} \zeta_{2}-\frac{11}{2} \zeta_{5}\right) \varepsilon^{2}+O\left(\varepsilon^{3}\right), \\
& S_{3,1}(\varepsilon)=\left(\frac{9}{2} \zeta_{5}-\zeta_{3} \zeta_{2}\right) \varepsilon+O\left(\varepsilon^{2}\right), \\
& S_{2,1,1}(\varepsilon)=\left(\frac{11}{2} \zeta_{5}-2 \zeta_{3} \zeta_{2}\right) \varepsilon+O\left(\varepsilon^{2}\right) .
\end{aligned}
$$

These relations can be obtained expanding the representation of the sums in terms of Mellin transforms of Nielsen integrals weighted by $1 /(1 \pm x)$. In case of the single harmonic sums the expansions result from Euler's $\psi$-function and its derivatives.

\section{B.8. Sample calculation for one of the sums}

In the following we illustrate the calculation of sum (B.60) in using the Sigma package:

$$
\sum_{j=1}^{\infty} \frac{1}{j+N} \sum_{i=1}^{\infty} \frac{S_{1}(i) S_{1}(i+j+N)}{i(i+j)}
$$

First, we treat the inner sum for $N$ fixed,

$$
F(j)=\sum_{i=1}^{\infty} \frac{S_{1}(i) S_{1}(i+j+N)}{i(i+j)} .
$$


By Sigma's creative telescoping algorithm we compute the recurrence relation

$$
\begin{aligned}
& -(j+N+1) j^{2} F(j)+(j+1)\left(3 j^{2}+3 N j+7 j+2 N+4\right) F(j+1) \\
& -(j+2)\left(3 j^{2}+3 N j+11 j+4 N+10\right) F(j+2) \\
& +(j+2)(j+3)(j+N+3) F(j+3) \\
& \quad=A(j)+B(j) S_{1}(j+N)
\end{aligned}
$$

where

$$
\begin{aligned}
A(j)= & \frac{1}{\left(j^{2}+3 j+2\right)\left(j^{2}+(2 N+3) j+N^{2}+3 N+2\right)^{2}} \\
& \times\left(3 j^{5}+(9 N+26) j^{4}+\left(10 N^{2}+63 N+86\right) j^{3}\right. \\
& +\left(5 N^{3}+49 N^{2}+156 N+137\right) j^{2}+\left(N^{4}+12 N^{3}+74 N^{2}+163 N+106\right) j \\
& \left.+5 N^{3}+34 N^{2}+61 N+32\right), \\
B(j)= & \frac{3 j^{3}+(4 N+13) j^{2}+\left(-N^{2}+11 N+18\right) j-N^{3}-2 N^{2}+7 N+8}{(j+1)(j+2)(j+N+1)(j+N+2)} .
\end{aligned}
$$

Next, we apply Sigma's recurrence solver and obtain three linearly independent solutions of the homogeneous version of the recurrence:

$$
\frac{1}{j}, \frac{S_{1}(j+N)}{j}, \quad \frac{-j S_{1}(j)+j S_{1}(j+N)+1}{j^{2}(N+1)}
$$

and one solution of the recurrence itself:

$$
\begin{aligned}
p(j)= & \frac{S_{1}(j+N)^{3}}{6 j}-\frac{S_{1}(j+N)^{2}}{2 j^{2}}+\left(-\frac{S_{2}(N)}{2 j}+\frac{S_{2}(j+N)}{2 j}+\frac{3}{N j+j}\right) S_{1}(j+N) \\
& -\frac{S_{3}(N)}{3 j}+\frac{S_{1}(N)^{2}}{2 N j+2 j}+\frac{3 N+2}{j^{2}(N+1)^{2}}+\frac{(j-2(N+1)) S_{1}(N)}{2 j^{2}(N+1)^{2}}+\frac{S_{2}(N)}{2 j^{2}} \\
& -\frac{S_{2}(j+N)}{2 j^{2}}+\frac{S_{3}(j+N)}{3 j}+\frac{\sum_{i=1}^{j} \frac{S_{1}(i+N)}{i^{2}}}{j}-\frac{\sum_{i=1}^{j} \frac{S_{1}(i+N)}{i}}{j^{2}} \\
& -\frac{\sum_{i=1}^{j} \frac{S_{1}(i) S_{1}(i+N)}{i}}{j}+\frac{\sum_{i=1}^{j} \frac{S_{2}(i+N)}{i}}{2 j}+\frac{\sum_{i=1}^{j} \frac{S_{1}(i+N)^{2}}{i}}{2 j} \\
& +S_{1}(j)\left(\frac{-3 N-2}{j(N+1)^{2}}+\frac{S_{1}(N)}{N j+j}-\frac{S_{2}(N)}{2 j}+\frac{\sum_{i=1}^{j} \frac{S_{1}(i+N)}{i}}{j}\right) \\
& -\frac{\sum_{i=1}^{j} \frac{S_{1}(i+N)}{(i+N)^{2}}}{j} .
\end{aligned}
$$

The function $F(j)$ is given by

$$
F(j)=a_{1} \frac{1}{j}+a_{2} \frac{S_{1}(j+N)}{j}+a_{3} \frac{-j S_{1}(j)+j S_{1}(j+N)+1}{j^{2}(N+1)}+p(j)
$$

for some properly chosen constants $a_{1}, a_{2}$ and $a_{3}$ which are free of $j$. Looking at the initial values for $j=1,2,3$ of $F(j)$ we can conclude that 


$$
\begin{aligned}
a_{1}= & -\frac{1}{6} S_{1}(N)^{3}-\frac{S_{1}(N)^{2}}{2 N+2}+\left(-\frac{1}{2} S_{2}(N)-\frac{1}{2(N+1)^{2}}\right) S_{1}(N) \\
& -S_{2,1}(N)+2 \zeta_{3} \\
a_{2}= & \frac{1}{2}\left(-S_{1}(N)^{2}-\frac{2 S_{1}(N)}{N+1}+S_{2}(N)+\frac{2\left((N+1)^{2} \zeta_{2}-1\right)}{(N+1)^{2}}\right), \\
a_{3}= & \frac{1}{2}(N+1) S_{1}(N)^{2}+S_{1}(N)-\frac{3 N+2}{N+1} .
\end{aligned}
$$

One obtains

$$
\begin{aligned}
F(j)= & -\frac{S_{1}(N)^{3}}{6 j}+\frac{S_{1}(N)^{2}}{2 j^{2}}+\left(\frac{1}{2 j N^{2}}-\frac{S_{2}(N)}{2 j}\right) S_{1}(N)+\frac{S_{1}(j+N)^{3}}{6 j} \\
& -\frac{S_{1}(j+N)^{2}}{2 j^{2}}+S_{1}(j)^{2}\left(-\frac{S_{1}(j+N)}{2 j}-\frac{1}{2 j N}\right)+\frac{S_{2}(N)}{2 j^{2}}-\frac{S_{2}(j+N)}{2 j^{2}} \\
& -\frac{S_{3}(N)}{3 j}+\frac{S_{3}(j+N)}{3 j}-\frac{\left.S_{2,1} N\right)}{j}+\frac{\sum_{i=1}^{j} \frac{S_{1}(i)^{2}}{i+N}}{2 j}+\frac{\sum_{i=1}^{j} \frac{S_{1}(i+N)}{i^{2}}}{2 j} \\
& -\frac{(j+N) \sum_{i=1}^{j} \frac{S_{1}(i+N)}{i}}{j^{2} N}-\frac{\sum_{i=1}^{j} \frac{S_{1}(i+N)}{(i+N)^{2}}}{j}+S_{1}(j)\left(-\frac{S_{1}(N)^{2}}{2 j}+\frac{S_{1}(j+N)}{j N}\right. \\
& \left.-\frac{S_{2}(N)}{2 j}+\frac{\sum_{i=1}^{j} \frac{S_{1}(i+N)}{i}}{j}+\frac{1}{2 j N^{2}}\right)+\frac{\sum_{i=1}^{j} \frac{S_{1}(i+N)^{2}}{i}}{2 j}+\frac{\sum_{i=1}^{j} \frac{S_{2}(i+N)}{i}}{2 j} \\
& +S_{1}(j+N)\left(\frac{\frac{S_{2}(j+N)}{N^{2}}-2 \zeta_{2}}{2 j}\right)+\frac{2 \zeta_{3}}{j} .
\end{aligned}
$$

Finally, we look at the indefinite nested sum

$$
S(N, a)=\sum_{j=1}^{a} \frac{F(j)}{j+N}
$$

with

$$
\lim _{a \rightarrow \infty} S(N, a)=\sum_{j=1}^{a} \sum_{j=1}^{\infty} \frac{S_{1}(i) S_{1}(i+j+N)}{i(i+j)(j+N)} .
$$

At this point we emphasize that the sum expression (B.82) with the derived sum representation of $F(j)$ fits into the input class of Sigma. Hence, we can apply Sigma's machinery again and arrive for $S(a, N)$ at the following sum representation

$$
\begin{aligned}
S(a, N)= & -\frac{S_{1}(N)^{4}}{8 N}+\frac{S_{1}(a+N) S_{1}(N)^{3}}{6 N}+\left(\frac{S_{2}(a)}{4 N}-\frac{S_{2}(N)}{4 N}+\frac{\zeta_{2}}{2 N}\right) S_{1}(N)^{2} \\
& +\left(S_{1}(a+N)\left(\frac{S_{2}(N)}{2 N}-\frac{1}{2 N^{3}}\right)-\frac{S_{2,1}(N)}{N}+\frac{2 \zeta_{3}}{N}\right) S_{1}(N)-\frac{S_{1}(a+N)^{4}}{24 N} \\
& +\frac{S_{2}(N)^{2}}{8 N}-\frac{S_{2}(a+N)^{2}}{8 N}+\frac{\left(\sum_{i=1}^{a} \frac{S_{1}(i+N)}{i}\right)^{2}}{2 N} \\
& +S_{1}(a)^{3}\left(-\frac{S_{1}(a+N)}{3 N}-\frac{1}{3 N^{2}}\right)+S_{1}(a)^{2}\left(-\frac{S_{1}(N)^{2}}{4 N}+\frac{S_{1}(a+N)}{2 N^{2}}\right.
\end{aligned}
$$




$$
\begin{aligned}
& \left.-\frac{S_{2}(N)}{4 N}+\frac{1}{4 N^{3}}\right)+S_{2}(a)\left(\frac{S_{2}(N)}{4 N}+\frac{1}{12 N^{3}}\right)+\frac{S_{3}(N)}{3 N^{2}}-\frac{S_{3}(a+N)}{3 N^{2}} \\
& +\frac{S_{4}(N)}{4 N}-\frac{S_{4}(a+N)}{4 N}-\frac{\sum_{i=1}^{a} \frac{S_{1}(i)}{(i+N)^{3}}}{3 N}-\frac{\sum_{i=1}^{a} \frac{S_{1}(i)^{2}}{(i+N)^{2}}}{2 N} \\
& +\left(\frac{S_{1}(a)}{2 N}-\frac{S_{1}(a+N)}{2 N}+\frac{1}{2 N^{2}}\right) \sum_{i=1}^{a} \frac{S_{1}(i)^{2}}{i+N}-\frac{\sum_{i=1}^{a} \frac{S_{1}(i)^{3}}{i+N}}{6 N}-\frac{\sum_{i=1}^{a} \frac{S_{1}(i+N)}{i^{3}}}{6 N} \\
& +\left(\frac{S_{1}(a)}{2 N}-\frac{S_{1}(a+N)}{2 N}\right) \sum_{i=1}^{a} \frac{S_{1}(i+N)}{i^{2}} \\
& +\left(-\frac{S_{1}(a)}{N}+\frac{S_{1}(a+N)}{N}+\frac{1}{N^{2}}\right) \sum_{i=1}^{a} \frac{S_{1}(i+N)}{(i+N)^{2}} \\
& +\frac{\sum_{i=1}^{a} \frac{S_{1}(i+N)}{(i+N)^{3}}}{N}-\frac{\sum_{i=1}^{a} \frac{S_{1}(i) S_{1}(i+N)}{i^{2}}}{2 N}+\frac{\sum_{i=1}^{a} \frac{S_{1}(i) S_{1}(i+N)}{(i+N)^{2}}}{N} \\
& +\frac{\sum_{i=1}^{a} \frac{S_{1}(i)^{2} S_{1}(i+N)}{i+N}}{N}+\left(\frac{S_{1}(a)}{2 N}-\frac{S_{1}(a+N)}{2 N}-\frac{1}{N^{2}}\right) \sum_{i=1}^{a} \frac{S_{1}(i+N)^{2}}{i} \\
& -\frac{\sum_{i=1}^{a} \frac{S_{1}(i+N)^{2}}{(i+N)^{2}}}{N}+\frac{2 \sum_{i=1}^{a} \frac{S_{1}(i+N)^{3}}{i}}{3 N}+\frac{\sum_{i=1}^{a} \frac{S_{1}(i+N) S_{2}(i)}{i}}{2 N} \\
& +\left(\frac{S_{1}(a)}{2 N}-\frac{S_{1}(a+N)}{2 N}\right) \sum_{i=1}^{a} \frac{S_{2}(i+N)}{i}+\frac{\sum_{i=1}^{a} \frac{S_{1}(i) S_{1}(i+N)^{2}}{i}}{2 N} \\
& -\frac{\sum_{i=1}^{a} \frac{S_{1}(i) S_{2}(i+N)}{i}}{2 N}+\frac{\sum_{i=1}^{a} \frac{S_{1}(i+N) S_{2}(i+N)}{i}}{N} \\
& +\frac{S_{2}(a+N)\left(1-3 N^{2} \zeta_{2}\right)}{6 N^{3}}+\frac{S_{2}(N)\left(3 N^{2} \zeta_{2}-1\right)}{6 N^{3}}+\frac{S_{1}(a+N)}{N^{2}} \\
& +\left(\sum_{i=1}^{a} \frac{S_{1}(i+N)}{i}\right)\left(\frac{S_{1}(a)^{2}}{2 N}+\left(-\frac{S_{1}(a+N)}{N}-\frac{1}{N^{2}}\right) S_{1}(a)-\frac{S_{1}(N)^{2}}{2 N}\right. \\
& \left.-\frac{S_{2}(a)}{2 N}-\frac{S_{2}(N)}{2 N}+\frac{\zeta_{2}}{N}-\frac{1}{2 N^{3}}\right)+S_{1}(a+N)^{2}\left(\frac{1-N^{2} \zeta_{2}}{2 N^{3}}-\frac{S_{2}(a+N)}{4 N}\right) \\
& +S_{1}(a+N)\left(\frac{S_{3}(N)}{3 N}-\frac{S_{3}(a+N)}{3 N}+\frac{S_{2,1}(N)}{N}-\frac{2 \zeta_{3}}{N}\right) \\
& +S_{1}(a)\left(-\frac{S_{1}(N)^{3}}{6 N}+\frac{S_{1}(a+N) S_{1}(N)^{2}}{2 N}+\left(\frac{1}{2 N^{3}}-\frac{S_{2}(N)}{2 N}\right) S_{1}(N)\right. \\
& +S_{1}(a+N)\left(\frac{S_{2}(N)}{2 N}-\frac{1}{2 N^{3}}\right)-\frac{S_{3}(N)}{3 N}+\frac{S_{3}(a+N)}{3 N} \\
& \left.-\frac{S_{2,1}(N)}{N}+\frac{2 \zeta_{3}}{N}\right)
\end{aligned}
$$

We remark that all the sums in this expression are algebraically independent, i.e., no relations occur that could cancel some of the involved sums. 


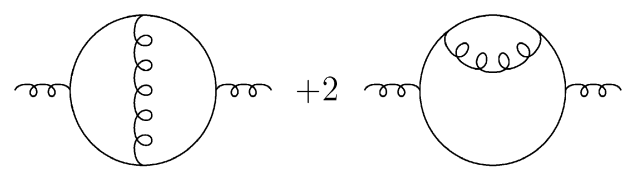

Fig. 3. Abelian part of the gluon self-energy due to heavy quarks.

Finally, we send $a$ to infinity in the last expression and note that the involved sum expressions can be simplified by the sum identities (B.37)-(B.51) and some additional identities of similar type. In the final expression divergences of the type $\sigma_{1}^{k}$ being contained in some of the terms vanish. We find the right-hand side of (B.60).

\section{Appendix C. The first moment of the operator matrix element}

After the analytic continuation from the even values of $N$ to $N \in \mathbf{C}$ is performed one may consider the limit $N \rightarrow 1$. In this procedure the term $\left(1+(-1)^{N}\right) / 2$ equals to 1 . At $O\left(a_{s}^{2}\right)$ the terms $\propto T_{F} C_{A}$ contain $1 / z$ contributions in momentum fraction space and their first moment diverges. For the other contributions to the un-renormalized operator matrix element (after mass renormalization to 2-loop order), the first moment is related to the Abelian part of the transverse contribution to the gluon propagator $\left.\Pi_{V}\left(p^{2}, m^{2}\right)\right|_{p^{2}=0}$, Fig. 3, except the term $\propto T_{F}^{2}$ which results from wave function renormalization. This was shown in [6] up to the constant term in $\varepsilon$. One obtains

$$
\Pi_{V}\left(p^{2}, m^{2}\right)=S_{\varepsilon} a_{s} T_{F} \Pi_{V}^{(1)}\left(p^{2}, m^{2}\right)+S_{\varepsilon}^{2} a_{s}^{2} C_{F} T_{F} \Pi_{V}^{(2)}\left(p^{2}, m^{2}\right)+O\left(a_{s}^{3}\right),
$$

with

$$
\begin{aligned}
& \lim _{p^{2} \rightarrow 0} \Pi_{V}^{(1)}\left(p^{2}, m^{2}\right)=\frac{1}{2} \hat{\hat{A}}_{Q g}^{(1), N=1}, \\
& \lim _{p^{2} \rightarrow 0} \Pi_{V}^{(2)}\left(p^{2}, m^{2}\right)=\left.\frac{1}{2} \hat{\hat{A}}_{Q g}^{(2), N=1}\right|_{C_{F}} .
\end{aligned}
$$

Here we extend the relation to the linear terms in $\varepsilon$. For the first moment the double pole contributions in $\varepsilon$ vanish in (C.2), (C.3). From the corresponding QED-expressions $\Pi_{T}^{V(k)}$ given in [64] by asymptotic expansion of the photon propagator $\left(1 / p^{2}\right) \tilde{\Pi}_{V}^{(k)}\left(p^{2}, m^{2}\right)$ in $m^{2} / p^{2}$ and adjusting the relative color factor for $k=2$ to $1 / 4=1 /\left(C_{F} C_{A}\right)$, due to the transition from QED to QCD, the comparison can be performed up to the constant term in $\varepsilon$. One obtains

$$
\begin{aligned}
& \lim _{p^{2} \rightarrow 0} \frac{1}{p^{2}} \tilde{\Pi}_{V}^{(1)}\left(p^{2}, m^{2}\right)=\frac{1}{2 T_{F}} \hat{\hat{A}}_{Q g}^{(1), N=1}=-\left(\frac{m^{2}}{\mu^{2}}\right)^{\varepsilon / 2} S_{\varepsilon}\left[\frac{8}{3 \varepsilon}+\frac{\varepsilon}{3} \zeta_{2}\right], \\
& \lim _{p^{2} \rightarrow 0} \frac{1}{p^{2}} \tilde{\Pi}_{V}^{(2)}\left(p^{2}, m^{2}\right) \\
& =\left.\frac{1}{2 T_{F} C_{F}} \hat{\hat{A}}_{Q g}^{(2), N=1}\right|_{C_{F}}=\left(\frac{m^{2}}{\mu^{2}}\right)^{\varepsilon}\left[-\frac{4}{\varepsilon}+15-\left(\frac{31}{4}+\zeta_{2}\right) \varepsilon\right]
\end{aligned}
$$

The latter term is easily obtained using MATAD [66]. 


\section{References}

[1] P.D. Thompson, J. Phys. G 34 (2007) N177, hep-ph/0703103.

[2] J. Blümlein, arXiv: 0706.2430 [hep-ph];

S. Bethke, Nucl. Phys. B (Proc. Suppl.) 135 (2004) 345, hep-ex/0407021.

[3] J. Blümlein, H. Böttcher, A. Guffanti, hep-ph/0607200.

[4] E. Laenen, S. Riemersma, J. Smith, W.L. van Neerven, Nucl. Phys. B 392 (1993) 162-229;

S. Riemersma, J. Smith, W.L. van Neerven, Phys. Lett. B 347 (1995) 143, hep-ph/9411431.

[5] S.I. Alekhin, J. Blümlein, Phys. Lett. B 594 (2004) 299, hep-ph/0404034.

[6] M. Buza, Y. Matiounine, J. Smith, R. Migneron, W.L. van Neerven, Nucl. Phys. B 472 (1996) 611, hep-ph/9601302.

[7] I. Bierenbaum, J. Blümlein, S. Klein, Nucl. Phys. B 780 (2007) 40, hep-ph/0703285.

[8] J. Blümlein, A. De Freitas, W.L. van Neerven, S. Klein, Nucl. Phys. B 755 (2006) 272, hep-ph/0608024.

[9] W. Furmanski, R. Petronzio, Z. Phys. C 11 (1982) 293, and references therein.

[10] W.L. van Neerven, E.B. Zijlstra, Phys. Lett. B 272 (1991) 127;

E.B. Zijlstra, W.L. van Neerven, Phys. Lett. B 273 (1991) 476;

E.B. Zijlstra, W.L. van Neerven, Nucl. Phys. B 383 (1992) 525;

S.A. Larin, J.A.M. Vermaseren, Z. Phys. C 57 (1993) 93;

S. Moch, J.A.M. Vermaseren, Nucl. Phys. B 573 (2000) 853, hep-ph/9912355.

[11] S.A. Larin, T. van Ritbergen, J.A.M. Vermaseren, Nucl. Phys. B 427 (1994) 41;

S.A. Larin, P. Nogueira, T. van Ritbergen, J.A.M. Vermaseren, Nucl. Phys. B 492 (1997) 338, hep-ph/9605317;

A. Retey, J.A.M. Vermaseren, Nucl. Phys. B 604 (2001) 281, hep-ph/0007294;

J. Blümlein, J.A.M. Vermaseren, Phys. Lett. B 606 (2005) 130, hep-ph/0411111;

J.A.M. Vermaseren, A. Vogt, S. Moch, Nucl. Phys. B 724 (2005) 3, hep-ph/0504242.

[12] E. Witten, Nucl. Phys. B 104 (1976) 445.

[13] J. Babcock, D.W. Sivers, S. Wolfram, Phys. Rev. D 18 (1978) 162;

M.A. Shifman, A.I. Vainshtein, V.I. Zakharov, Nucl. Phys. B 136 (1978) 157, Yad. Fiz. 27 (1978) 455;

J.P. Leveille, T.J. Weiler, Nucl. Phys. B 147 (1979) 147;

M. Glück, E. Hoffmann, E. Reya, Z. Phys. C 13 (1982) 119.

[14] Y. Matiounine, J. Smith, W.L. van Neerven, Phys. Rev. D 57 (1998) 6701, hep-ph/9801224.

[15] Y. Matiounine, J. Smith, W.L. van Neerven, Phys. Rev. D 58 (1998) 076002, hep-ph/9803439.

[16] J.A.M. Vermaseren, Int. J. Mod. Phys. A 14 (1999) 2037, hep-ph/9806280.

[17] S. Weinzierl, Comput. Phys. Commun. 145 (2002) 357, math-ph/0201011.

[18] S.-O. Moch, P. Uwer, Comput. Phys. Commun. 174 (2006) 759, math-ph/0508008.

[19] C. Schneider, Ann. Comb. 9 (1) (2005) 75;

C. Schneider, in: Proceedings of ISSAC'05, ACM Press, 2005, p. 285;

C. Schneider, in: Proceedings of FPSAC'07, 2007, p. 1;

C. Schneider, J. Difference Equ. Appl. 11 (9) (2005) 799;

C. Schneider, J. Algebra Appl. 6 (3) (2007) 415;

C. Schneider, Sém. Lothar. Combin. 56 (2007), Article B56b;

C. Schneider, Habilitation Thesis, JKU Linz, 2007.

[20] J. Blümlein, S. Kurth, Phys. Rev. D 60 (1999) 014018, hep-ph/9810241.

[21] F.J. Yndurain, The Theory of Quark and Gluon Interactions, Springer, Berlin, 1983;

R. Hamberg, Second order gluonic contributions to physical quantities, PhD Thesis, Leiden University, Leiden, 1991.

[22] S.D. Drell, T.M. Yan, Ann. Phys. 66 (1971) 578, Ann. Phys. 281 (2000) 450.

[23] E. Fermi, Z. Phys. 29 (1924) 315.

[24] D.J. Gross, F. Wilczek, Phys. Rev. D 8 (1973) 3633.

[25] D.J. Gross, F. Wilczek, Phys. Rev. D 9 (1974) 980;

H. Georgi, H.D. Politzer, Phys. Rev. D 9 (1974) 416.

[26] J. Blümlein, H. Böttcher, arXiv: 0802.0408 [hep-ph].

[27] H. Georgi, H.D. Politzer, Phys. Rev. D 14 (1976) 1829.

[28] M. Glück, E. Reya, Mod. Phys. Lett. A 22 (2007) 351, hep-ph/0608276.

[29] M. Buza, Y. Matiounine, J. Smith, W.L. van Neerven, Eur. Phys. J. C 1 (1998) 301, hep-ph/9612398.

[30] I. Bierenbaum, J. Blümlein, S. Klein, in preparation.

[31] C.G. Callan, Phys. Rev. D 2 (1970) 1541;

K. Symanzik, Commun. Math. Phys. 18 (1970) 227. 
[32] J. Blümlein, Eur. Phys. J. C 20 (2001) 683, hep-ph/0104099.

[33] K. Symanzik, Commun. Math. Phys. 23 (1971) 49;

C.G. Callan, Phys. Rev. D 5 (1972) 3202;

N.H. Christ, B. Hasslacher, A.H. Mueller, Phys. Rev. D 6 (1972) 3543.

[34] D.J. Gross, F. Wilczek, Phys. Rev. Lett. 30 (1973) 1343;

H.D. Politzer, Phys. Rev. Lett. 30 (1973) 1346;

G. 't Hooft, unpublished;

W.E. Caswell, Phys. Rev. Lett. 33 (1974) 244;

D.R.T. Jones, Nucl. Phys. B 75 (1974) 531.

[35] W. Celmaster, R.J. Gonsalves, Phys. Rev. Lett. 42 (1979) 1435;

W. Celmaster, R.J. Gonsalves, Phys. Rev. D 20 (1979) 1420.

[36] L.F. Abbott, Nucl. Phys. B 185 (1981) 189.

[37] A. Rebhan, Z. Phys. C 30 (1986) 309;

F. Jegerlehner, O.V. Tarasov, Nucl. Phys. B 549 (1999) 481, hep-ph/9809485.

[38] O. Nachtmann, W. Wetzel, Nucl. Phys. B 146 (1978) 273;

B.J. Edwards, T.D. Gottschalk, Nucl. Phys. B 196 (1982) 328.

[39] J.C. Collins, F. Wilczek, A. Zee, Phys. Rev. D 18 (1978) 242;

B.A. Ovrut, H.J. Schnitzer, Nucl. Phys. B 179 (1981) 381;

B.A. Ovrut, H.J. Schnitzer, Nucl. Phys. B 189 (1981) 509;

W. Bernreuther, W. Wetzel, Nucl. Phys. B 197 (1982) 228;

W. Bernreuther, W. Wetzel, Nucl. Phys. B 513 (1998) 758, Erratum;

W. Bernreuther, Ann. Phys. 151 (1983) 127.

[40] R. Tarrach, Nucl. Phys. B 183 (1981) 384;

O. Nachtmann, W. Wetzel, Nucl. Phys. B 187 (1981) 333.

[41] N. Gray, D.J. Broadhurst, W. Grafe, K. Schilcher, Z. Phys. C 48 (1990) 673;

D.J. Broadhurst, N. Gray, K. Schilcher, Z. Phys. C 52 (1991) 111.

[42] J. Fleischer, F. Jegerlehner, O.V. Tarasov, O.L. Veretin, Nucl. Phys. B 539 (1999) 671, hep-ph/9803493; J. Fleischer, F. Jegerlehner, O.V. Tarasov, O.L. Veretin, Nucl. Phys. B 571 (2000) 511, Erratum.

[43] K.G. Chetyrkin, M. Steinhauser, Nucl. Phys. B 573 (2000) 617, hep-ph/9911434.

[44] J. Lagrange, Nouvelles recherches sur la nature et la propagation du son, Miscellanea Taurinensis, t. II, 1760-1761; Oeuvres t. I, p. 263;

C.F. Gauss, Theoria attractionis corporum sphaeroidicorum ellipticorum homogeneorum methodo novo tractate,

Commentationes societas scientiarum Gottingensis recentiores, vol. III, 1813, Werke Bd. V, pp. 5-7;

G. Green, Essay on the mathematical theory of electricity and magnetism, Nottingham, 1828, Green Papers, pp. 1-115;

M. Ostrogradski, Mem. Ac. Sci. St. Peters 6 (1831) 39;

K.G. Chetyrkin, A.L. Kataev, F.V. Tkachov, Nucl. Phys. B 174 (1980) 345.

[45] W.N. Bailey, Generalized Hypergeometric Series, Cambridge Univ. Press, Cambridge, 1935;

L.J. Slater, Generalized Hypergeometric Functions, Cambridge Univ. Press, Cambridge, 1966.

[46] C. Schneider, J. Symbolic Comput. (2008), doi:10.1016/j.jsc.2008.01.001.

[47] M. Karr, J. Assoc. Comput. Mach. 28 (1981) 305.

[48] A.B. Goncharov, Math. Res. Lett. 5 (1998) 497.

[49] J.M. Borwein, D.M. Bradley, D.J. Broadhurst, P. Lisonek, Trans. Am. Math. Soc. 353 (2001) 907, math/9910045.

[50] Hoang Ngoc Minh, M. Petitot, J. van der Hoeven, Discrete Math. 225 (2000) 217.

[51] S. Moch, P. Uwer, S. Weinzierl, J. Math. Phys. 43 (2002) 3363, hep-ph/0110083.

[52] M. Petkovšek, H.S. Wilf, D. Zeilberger, $A=B$, A.K. Peters, Wellesley, MA, 1996;

D. Zeilberger, J. Symbolic Comput. 11 (1991) 195.

[53] I. Bierenbaum, J. Blümlein, S. Klein, C. Schneider, arXiv: 0707.4659 [math-ph].

[54] J.A.M. Vermaseren, math-ph/0010025.

[55] J. Blümlein, Comput. Phys. Commun. 159 (2004) 19, hep-ph/0311046.

[56] J. Blümlein, DESY 07-042.

[57] J. Blümlein, Comput. Phys. Commun. 133 (2000) 76, hep-ph/0003100; J. Blümlein, S.O. Moch, Phys. Lett. B 614 (2005) 53, hep-ph/0503188.

[58] J. Blümlein, A. Vogt, Phys. Rev. D 58 (1998) 014020, hep-ph/9712546.

[59] E.W. Barnes, Proc. London Math. Soc. (2) 6 (1908) 141;

E.W. Barnes, Quart. J. Math. 41 (1910) 136. 
[60] H. Mellin, Math. Ann. 68 (1910) 305;

E.T. Whittaker, G.N. Watson, A Course of Modern Analysis, Cambridge Univ. Press, Cambridge, 1927, reprinted 1996;

E.C. Titchmarsh, Introduction to the Theory of Fourier Integrals, Calendron Press, Oxford, 1937, second edition 1948.

[61] I. Bierenbaum, S. Weinzierl, Eur. Phys. J. C 32 (2003) 67, hep-ph/0308311.

[62] I. Bierenbaum, J. Blümlein, S. Klein, Nucl. Phys. B (Proc. Suppl.) 160 (2006) 85, hep-ph/0607300;

I. Bierenbaum, J. Blumlein, S. Klein, Nucl. Phys. B (Proc. Suppl.) 174 (2007) 75;

I. Bierenbaum, J. Blumlein, S. Klein, Phys. Lett. B 648 (2007) 195, hep-ph/0702265.

[63] M. Czakon, Comput. Phys. Commun. 175 (2006) 559, hep-ph/0511200.

[64] A. Djouadi, P. Gambino, Phys. Rev. D 49 (1994) 3499, hep-ph/9309298;

A. Djouadi, P. Gambino, Phys. Rev. D 53 (1996) 4111, Erratum.

[65] J. Blümlein, Nucl. Phys. B (Proc. Suppl.) 135 (2004) 225, hep-ph/0407044;

J. Blümlein, V. Ravindran, Nucl. Phys. B 749 (2006) 1, hep-ph/0604019;

J. Blümlein, V. Ravindran, Nucl. Phys. B 716 (2005) 128, hep-ph/0501178;

M. Dittmar, et al., hep-ph/0511119;

J. Blümlein, S. Moch, in preparation;

J. Blümlein, S. Klein, arXiv: 0706.2426 [hep-ph].

[66] M. Steinhauser, Comput. Phys. Commun. 134 (2001) 335, hep-ph/0009029.

[67] J.A.M. Vermaseren, Comput. Phys. Commun. 83 (1994) 45. 